\title{
Willingness to pay for and acceptance of cervical cancer prevention methods: A systematic review and meta-analysis
}

\author{
Anahita Shokri Jamnani ${ }^{1}$, Aziz Rezapour ${ }^{2} * \mathbb{D}$, Najmeh Moradi $^{3}$, Mostafa Langarizadeh $^{4}$ \\ Received: 18 Aug 2020 \\ Published: 23 Jun 2021
}

\section{Abstract}

Background: The Willingness to pay (WTP) for and acceptance of cervical cancer prevention (CCP) methods have an important role in the control of this type of cancer. Therefore, the aim of this study was to estimate the WTP and acceptance of CCP methods with the contingent valuation method (CVM).

Methods: In this systematic review and meta-analysis study, the required information was collected by searching relevant keywords in PubMed, Scopus, Embase, Web of Knowledge, and their Persian equivalent in the Scientific Information Database (SID) and Elmnet databases during January 1, 2000 to June 30, 2020. All studies that reported the WTP and CCP methods with the CVM in English or Persian were included. The reporting quality of studies was assessed by strengthening the Reporting of Observational Studies in Epidemiology (STROBE). Comprehensive meta-analysis (CMA: 2) software was used to conduct the meta-analysis. The content analysis method was used for qualitative data analysis.

Results: Finally, 28 articles (with 49610 people) were included in the study. Most of the participants were women (35.7\%). The HPV vaccine was the most common method of prevention (75\%). The overall acceptance rate was $64 \%$ and the overall positive WTP rate was $66 \%$. The average WTP was US $\$ 30.44$, which accounts for about $0.84 \%$ of GDP per capita. The most significant effective factors included income, age, education, high-risk sexual behaviors, and awareness of cervical cancer, belief in the risk of cervical cancer, and belief about the effectiveness of prevention methods. The cost was the most important reason for the unwillingness to pay and accept.

Conclusion: Results show that the WTP and acceptance rate of CCP methods are relatively high. It is recommended to reduce the cost of prevention methods, especially the HPV vaccine, and to increase awareness and improve the attitude of people. Also, it is recommended to consider other methods of estimation of WTP and other cancers in future studies.

Keywords: Acceptance, Preventive Measures, Cervical Cancer, Human Papillomavirus Vaccine

Conflicts of Interest: None

Funding: Iran University of Medical Sciences (Grant No: IUMS/SHMIS_98-2-37-15495)

\section{*This work has been published under CC BY-NC-SA 1.0 license.}

Copyright $₫$ Iran University of Medical Sciences

Cite this article as: Shokri Jamnani A, Rezapour A, Moradi N, Langarizadeh M. Willingness to pay for and acceptance of cervical cancer prevention methods: A systematic review and meta-analysis. Med J Islam Repub Iran. 2021 (23 Jun);35:81. https://doi.org/10.47176/mjiri.35.81

\section{Introduction}

Over the past 2 decades, with the expansion of primary

Corresponding author: Dr Aziz Rezapour, Rezapour.a@iums.ac.ir

1. Department of Health Economics, School of Health Management and Information Sciences, Iran University of Medical Sciences, Tehran, Iran

2. Health Management and Economics Research Center, School of Health Management and Information Sciences, Iran University of Medical Sciences, Tehran, Iran

3. Health Management and Economics Research Center, Iran University of Medical Sciences, Tehran, Iran

4. Department of Health Information Management, School of Health Management and Information Sciences, Iran University of Medical Sciences, Tehran, Iran health care, success in controlling infectious diseases,

$\uparrow$ What is "already known" in this topic:

In recent years, many studies have been conducted on the willingness to pay (WTP) and acceptance rate of cervical cancer prevention (CCP) methods. However, based on the results of reviewing the literature, the results of these studies were not systematically collected and analyzed.

\section{$\rightarrow$ What this article adds:}

According to the results of this study, it is recommended that attentions should be paid to reducing the cost of preventive measures, focusing on target age groups; and reducing risky sexual behaviors to increase the WTP and acceptance of CCP methods. 
covering more than $90 \%$ of the population, and changing population structure has increased the prevalence of noncommunicable diseases such as cancer (1). Cancers are now one of the major problems of health systems around the world (2). More than $70 \%$ of all cancer-related deaths occur in low- and middle-income countries (LMICs). Mathers and Loncar (2006) results showed that global cancer deaths will be increase, with 11.5 million deaths in 2030 (3). Today, cancers are plaguing these countries with increasing attenuation in LMICs as well as in high-income countries (HICs).

One of the most important types of cancers is cervical cancer, which is the second leading cause of mortality for women after breast cancer at the age of 15 to 44 years. Even in many LMICs, cervical cancer is the most common cause of cancer deaths, with half a million new cases diagnosed worldwide each year, with nearly 250000 deaths (4). Relatively effective methods are available today in the management of this cancer (5).

Many different tests and methods have been developed for the early detection and prevention of cervical cancer (6). Most of these methods are highly effective and are among the simple, low-cost, painless, and high- effectiveness prevention methods (7).

Despite these benefits of cervical cancer prevention (CCP) methods, the results of the previous studies show that these methods are not well-liked and accepted. Also, for a variety of reasons, including financial problems, people have a little willingness to pay (WTP) for these methods (8-10).

In economics, the WTP is the highest price a person is willing to pay for a unit more than a service or good (11). Various methods have been used to measure WTP; one of the most common and widely used methods is the contingent valuation method (CVM). In this method, people are asked to state the maximum amount of money that they are willing to pay for a particular product that may not be purchased (nonconsumable). In other words, this method specifies the maximum price that a person is WTP to buy a product or receive health services (12).

In recent years, especially with the production and use of human papillomavirus (HPV) vaccines, many studies have been conducted on the WTP and acceptance rate of CCP methods (13-15). However, the results of these studies were not systematically collected and analyzed. The availability of such information is critical for effective decision-making and planning to help implement or develop cancer prevention programs. Therefore, the purpose of this study was to estimate the WTP and the acceptance of different methods of CCP using the CVM method.

\section{Methods}

This systematic review and meta-analysis study was conducted in 2020. In this study, authors used the systematic review guide book entitled "Systematic Review to Support Evidence-Based Medicine" (16) and the preferred reporting items for systematic reviews and meta-analyses (PRISMA) statement (17).

\section{Search Strategy}

The search strategy in this study was developed and implemented by an experienced and knowledgeable librarian with the guidance of an expert in the subject area (Appendix 1).

The required information was collected by searching relevant keywords and medical subject heading (Mesh) terms in 4 subjects in PubMed, Scopus, Embase, Web of Knowledge, and their Persian equivalent have been searched in the scientific information database (SID) and Elmnet database.

The timeframe selected for searching the articles was January 1, 2000 to June 30, 2020. To identify and cover most articles published after searching the databases, some prestigious journals (the European Journal of Cancer Prevention, Cancer Prevention Research, the Asian Pacific Journal of Cancer Prevention, Cancer Communications, BMC Cancer, Cancer Epidemiology, the International Journal of Cancer Management, etc.) in the field of study and the Google Scholar search engine were also searched manually. After excluding studies that had little relevance to the study objectives, to increase the certainty of identifying and reviewing existing studies, reference checks of selected articles, Gray literature review (European Association for Grey Literature Exploitation (EAGLE), and Health Care Management Information Consortium (HMIC)) was done. Finally, all relevant articles were collected by contacting an expert.

\section{Inclusion and Exclusion Criteria}

All studies that reported the WTP and CCP methods with the CVM in English or P'ersian were included in the study.

\section{Evaluation of the Reports on the Quality of Articles}

The quality of reporting of included studies was evaluated by 2 independent reviewers using the Strengthening the Reporting of Observational Studies in Epidemiology (STROBE) checklist (18). In this study, minor modifications were made to the questions, and finally, 33 items were measured. The checklist options included "Yes", "No", "Not Applicable" and "Unclear". Cases of disagreement between the reviewers were referred to a third person who had more knowledge and experience in the methodological issues of the research.

\section{Data Extraction}

To extract data, 2 data extraction forms were designed in Word: 2013 software (one for extracting general specifications of studies and the other for extracting studies results). The general profile form of studies included author, year, country, the purpose of study, study design, participants: $\mathrm{N}$ (response rate), data collection tool, type of test, administration, type of questions and type of information. Information on the results of the study form included the demographic characteristics of participants, $\%$ of acceptance of the test, $\%$ of positive WTP, WTP (US\$) mean \pm SD OR median (CR), WTP as a \% of gross domestic product (GDP) per capita, other results of variable effects in WTP (significant positive, significant 
negative, and nonsignificant) were the main reasons for no WTP (\%). Initially, the data of 5 papers were extracted experimentally and the shortcomings and problems in the original form were eliminated.

\section{Data Analysis Methods}

A meta-analysis was used to estimate the quantitative data (acceptance of CCP methods, WTP for CCP methods, and percentage of WTP per capita GDP). CMA: 2 (Comprehensive Meta-Analysis) software was used to perform the meta-analysis with a random effect model. Forest plot diagrams were used to report the results. Q and $\mathrm{I}^{2}$ tests were used to measure the heterogeneity of the results. In this study, with $\mathrm{I}^{2}$ more than $50 \%$, the heterogeneity of articles was determined.

To calculate the WTP, the countries' currency value reported in studies was converted to US dollars (in study published year) using a free online website (19). In cases where the year of the study was not reported, the baseline year was considered 1 year before the study was published. In some studies, the values were not reported as central indexes (mean or median) indices (were reported as intervals), in which case the intermediate interval was used as the WTP. In some studies, the values were reported as a percentage of the participants (eg, 65\% of participants have X amount of WTP); in such cases, the reported amount was chosen as the baseline. Also, to calculate the percentage of WTP from GDP per capita, the amount of WTP was divided by GDP per capita in the year of the study or 1 year before the study was published. The World Bank data were used as a basis for calculating GDP per capita and country segmentation based on economic status (20).

Given how the results were reported on the monetary amounts (in US dollars) that individuals were willing to pay, the researchers decided to convert the reported values in mean \pm SD to mean (95\% confidence interval) format using the $\mathrm{Z}$ statistics method by online tools. Studies that

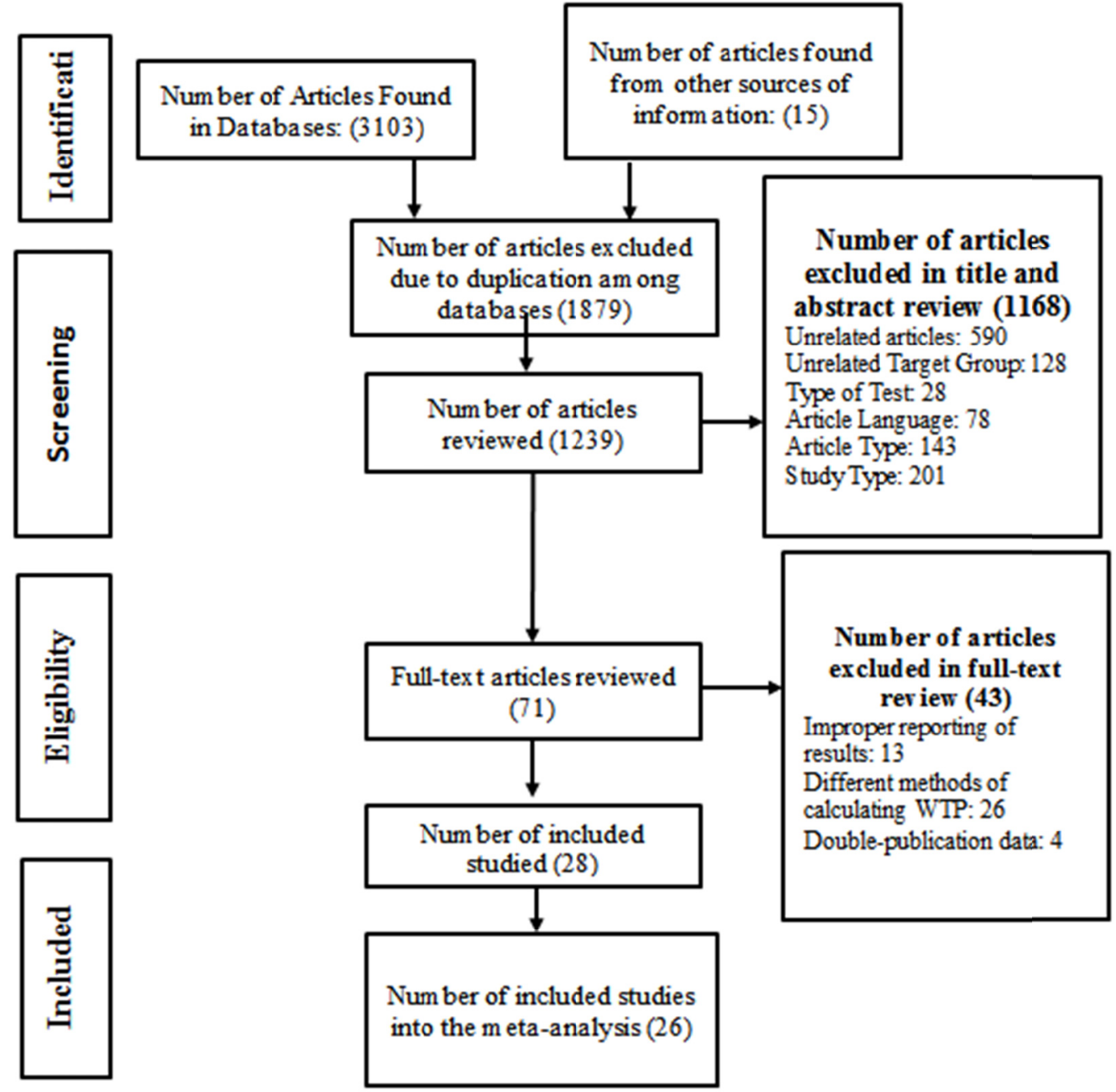

Fig. 1. Search and screening process for articles 
reported the median or only 1 number (without SD or CI) were not included in the meta-analysis.

Content-analysis methods were used for qualitative data analysis, which is a method for identifying, analyzing, and reporting patterns within themes and is widely used in qualitative data analysis (21). Data were analyzed by 2 researchers. The steps for analyzing and coding the data were as follows: familiarity with the content of the articles (reading multiple times extracted data); identifying and extracting primary codes (identifying and extracting data more relevant to the primary codes); identifying themes (Inserting extracted primary codes into related themes); reviewing and completing identified themes; naming and defining themes; and ensuring the reliability of the extracted codes and themes (agreeing between the 2 coders through discussion and fixing Disputes).

\section{Results}

Out of the 3118 articles found from the databases and other sources, 1879 were excluded due to duplication between databases. In the title and abstract screening phase, 1168 cases were also excluded. A total of 43 studies were excluded from the full-text review, and finally, 28 articles were included in the study, of which 26 were included in the meta-analysis (Fig. 1).

The characteristics and results of the reviewed articles are presented in (Tables 1 and 2).

\section{Characteristics of the Included Studies}

Studies were conducted in 19 different countries (Scotland, Hong Kong, Malaysia, UK, South Africa, Thailand, Argentina, Vietnam, Nigeria, Taiwan, USA, Sweden, Korea, Cambodia, Ghana, Ethiopia, China, Indonesia, and Tanzania). Most studies were conducted in upper-middle- income economies (UMIEs) (based on New World Bank country classifications by income level: 2019-20 (46)).

\section{Acceptance of Cervical Cancer Prevention Methods}

Out of the 28 articles included in the study, 17 studies (23 data) were meta-analysis on the rate of acceptance of CCP methods. The results showed that the overall acceptance rate was 64\% (95\% C'I, 56-72) (Fig. 2).

The results also showed that among the different groups, the highest acceptance was among women (78\%); based on the economic status of countries, the highest acceptance was in LIEs (88\%); and based on the type of testing, the highest acceptance was related to overall prevention services (89.5) (Table 3).

The results of the heterogeneous assessment also showed that the results of the studies had a relatively acceptable heterogeneity $\left(\mathrm{Q}=193.8 ; \mathrm{df}=22 ; \mathrm{I}^{2}=88.6 ; \mathrm{p}<.001\right)$.

\section{Willingness to Pay for Cervical Cancer Prevention} Methods

Out of the 28 articles included in the study, 19 studies (22 data) were meta-analysis on WTP for CCP methods. The results showed that the overall positive WTP rate was $66 \%$ (95\% CI; 57-76) (Fig. 3).

The results also showed that among the different groups, the highest rate of positive WTP was found among students and other groups, and according to the economic status of countries, the highest rate of Positive WTP was in the low-income economies (LIEs). According to the type of prevention method, the highest WTP is for the Pap smear test (Table 3). The results of the heterogeneous assessment showed that the results of the studies have a relatively high heterogeneity $(Q=226 ; D F=21$; $\mathrm{I}^{2}=90.7 ; \mathrm{p}<0.001$ ).
Study name

Wordsworth $\mathrm{S}$ et al, 2001

Choi HCW et al, 2013 (1)

Choi HCW et al, 2013 (2)

Choi HCW et al, 2013 (3)

Rajiah $\mathrm{K}$ et al, 2015

Hoque ME et al, 2013

Maharajan MK et al, 2015

Kruiroongroj $\mathrm{S}$ et al, 2014 (1)

Kruiroongroj $\mathrm{S}$ et al, 2014 (2)

Songthap A et al, 2012 (1)

Songthap A et al, 2012 (2)

Songthap A et al, 2012 (3)

Alder $S$ et al, 2015

Umeh IB et al, 2016

Yan Yuen Wvv et al, 2018 (1)

Yan Yuen Wwv et al, 2018 (2)

Dahlström LA et al,2010

Touch S and Oh JK, 2018

Opoku CA et al, 2016

Lin $Y$ et al, 2020

You $\mathrm{D}$ et $\mathrm{al}, 2020$

Lin $W$ et al, 2020

Weng $Q$ et al, 2020

Event
rate
0.089
0.028
0.038
0.027
0.084
0.077
0.090
0.077
0.074
0.026
0.045
0.043
0.090
0.093
0.085
0.087
0.076
0.062
0.097
0.059
0.054
0.063
0.088
0.064

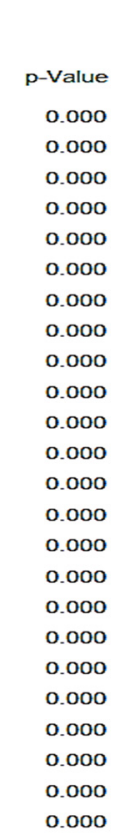

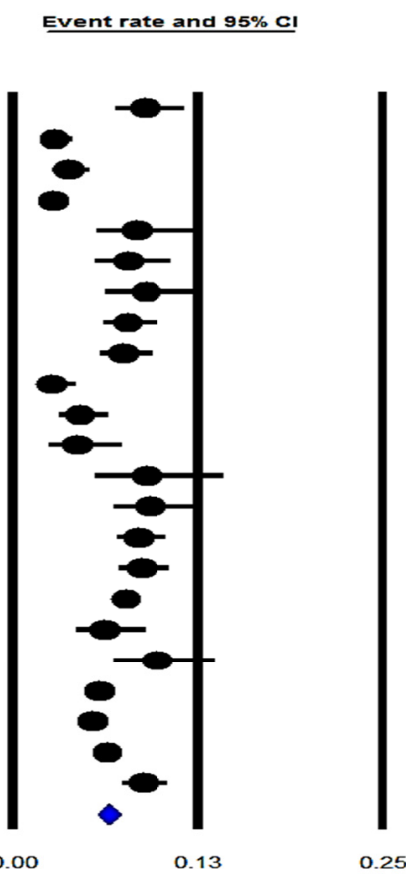

Fig. 2. Meta-analysis of Overall Rate of Acceptance of Cervical Cancer Prevention Methods Based on a Randonn Effect Model with 95\% Confidence Level 


\begin{tabular}{|c|c|c|c|c|c|c|c|c|c|}
\hline Author, year country & Aim of study & $\begin{array}{l}\text { Study } \\
\text { design }\end{array}$ & $\begin{array}{l}\text { Participants: N } \\
\text { (response rate) }\end{array}$ & $\begin{array}{l}\text { Data collection } \\
\text { tool }\end{array}$ & $\begin{array}{l}\text { Type of } \\
\text { prevention } \\
\text { methods }\end{array}$ & Administration & Type of questions & $\begin{array}{l}\text { Informed by } \\
\ldots \text { information }\end{array}$ & Type of information \\
\hline $\begin{array}{l}\text { 1. Wordsworth S, et al: } \\
\text { 2001: Scotland (22) }\end{array}$ & $\begin{array}{l}\text { Assess the value of the } \\
\text { cervical smear test to } \\
\text { women, }\end{array}$ & $\begin{array}{l}\text { A Postal } \\
\text { Survey }\end{array}$ & $\begin{array}{l}\text { Women aged 20-59: } \\
595(30)\end{array}$ & Questionnaire & Pap-smear & Mailed & $\begin{array}{l}\text { Payment card } \\
\text { technique }\end{array}$ & General & $\begin{array}{c}\text { Time of cervical } \\
\text { smear, method of } \\
\text { carrying out screening, } \\
\text { time between smears } \\
\text { time for results, } \\
\text { chance of being re- } \\
\text { called, chance of hav- } \\
\text { ing an abnormality, } \\
\text { chance of dying hav- } \\
\text { ing an abnormality } \\
\text { will result in a need } \\
\text { for further smears }\end{array}$ \\
\hline $\begin{array}{l}\text { 2. Choi HCW et al, 2013: } \\
\text { Hong Kong (23) }\end{array}$ & $\begin{array}{l}\text { Provide a more repre- } \\
\text { sentative and updated } \\
\text { assessment on the } \\
\text { acceptability of female } \\
\text { adoles- cent HPVvac- } \\
\text { cination }\end{array}$ & Survey & $\begin{array}{c}\text { Mothers with daugh- } \\
\text { ters } \\
\text { aged } \leq 18 \text { years' in } \\
2008 \text { year: } 1022(39.3) \\
\text { In } 2012 \text { year: } 1005 \\
(50.2) \\
\text { Adolescent schoolgirl: } \\
2252 \text { ( } 93.4)\end{array}$ & $\begin{array}{l}\text { Random digit- } \\
\text { dialing tele- } \\
\text { phone inter- } \\
\text { viewing }\end{array}$ & HPV vaccination & $\begin{array}{l}\text { Telephone inter- } \\
\text { viewing }\end{array}$ & Open ended & General & $\begin{array}{l}\text { HPVvaccineand } \\
\text { its market price range }\end{array}$ \\
\hline $\begin{array}{l}\text { 3. Rajiah K et al, 2015: } \\
\text { Malaysia (18) }\end{array}$ & $\begin{array}{l}\text { Evaluate the } \\
\text { knowledge, attitude, } \\
\text { practice and to find out } \\
\text { the willingness to pay } \\
\text { for HPV vaccination }\end{array}$ & $\begin{array}{l}\text { Cross- } \\
\text { Sectional } \\
\text { Study }\end{array}$ & $\begin{array}{l}\text { University students } \\
\text { studying health sci- } \\
\text { ences: } 273(85.3)\end{array}$ & $\begin{array}{c}\text { Self- } \\
\text { administered } \\
\text { validated ques- } \\
\text { tionnaire }\end{array}$ & HPV vaccination & $\begin{array}{l}\text { Telephone inter- } \\
\text { viewing }\end{array}$ & - & - & - \\
\hline $\begin{array}{l}\text { 4. Tarekegn AA, et al, } \\
\text { 2019: Ethiopia (24) }\end{array}$ & $\begin{array}{l}\text { Willingness to pay and } \\
\text { associated factors for } \\
\text { cervical cancer screen- } \\
\text { ing program }\end{array}$ & $\begin{array}{l}\text { Cross- } \\
\text { Sectional } \\
\text { Study }\end{array}$ & $\begin{array}{l}\text { Female health profes- } \\
\text { sionals in the College } \\
\text { of Medicine and } \\
\text { Health Sciences: } 392 \\
(92.7)\end{array}$ & Questionnaire & Screening service & Face-to-face & $\begin{array}{l}\text { Double- } \\
\text { Bounded Dichot- } \\
\text { omous Choice }\end{array}$ & - & - \\
\hline $\begin{array}{l}\text { 5. Tarekegn AA and } \\
\text { Yismaw AE, 2019: Ethi- } \\
\text { opia (25) }\end{array}$ & $\begin{array}{l}\text { Willingness to accept } \\
\text { and pay, and associated } \\
\text { factors for human pap- } \\
\text { illoma virus vaccina- } \\
\text { tion }\end{array}$ & $\begin{array}{l}\text { Cross- } \\
\text { Sectional } \\
\text { Study }\end{array}$ & $\begin{array}{c}\text { Female health profes- } \\
\text { sionals in the College } \\
\text { of Medicine and } \\
\text { Health Sciences: } 392 \\
\text { (92.7) }\end{array}$ & Questionnaire & HPV vaccination & Face-to-face & $\begin{array}{l}\text { Double- } \\
\text { Bounded Dichot- } \\
\text { omous Choice }\end{array}$ & - & - \\
\hline
\end{tabular}




\begin{tabular}{|c|c|c|c|c|c|c|c|c|c|}
\hline Author, year country & Aim of study & $\begin{array}{l}\text { Study } \\
\text { design }\end{array}$ & $\begin{array}{l}\text { Participants: } \mathrm{N} \\
\text { (response rate) }\end{array}$ & $\begin{array}{l}\text { Data collection } \\
\text { tool }\end{array}$ & $\begin{array}{l}\text { Type of } \\
\text { prevention } \\
\text { methods }\end{array}$ & Administration & $\begin{array}{c}\text { Type of } \\
\text { questions }\end{array}$ & $\begin{array}{l}\text { Informed by } \\
\ldots . \text { information }\end{array}$ & Type of information \\
\hline $\begin{array}{l}\text { 6. Philips Z,et al, 2003: } \\
\text { UK (17) }\end{array}$ & $\begin{array}{c}\text { Delineate } \\
\text { their knowledge of } \\
\text { cervical cancer and } \\
\text { screening and valua- } \\
\text { tion } \\
\text { of the introduction of } \\
\text { HPV testing }\end{array}$ & $\begin{array}{l}\text { Cross- } \\
\text { Sectional } \\
\text { Study }\end{array}$ & $\begin{array}{c}\text { female students at } \\
\text { the University of } \\
\text { Nottingham: } 222 \\
\text { (44.4) }\end{array}$ & Questionnaire & HPV vaccination & Mailed & $\begin{array}{l}\text { payment card } \\
\text { technique }\end{array}$ & - & - \\
\hline $\begin{array}{l}\text { 7. Hoque ME et al, 2013: } \\
\text { South Africa (26) }\end{array}$ & $\begin{array}{l}\text { Assess the awareness } \\
\text { of CC and its risk } \\
\text { factors and to deter- } \\
\text { mine the level of } \\
\text { acceptability of HPV } \\
\text { vaccination }\end{array}$ & $\begin{array}{l}\text { Cross- } \\
\text { Sectional } \\
\text { Study }\end{array}$ & $\begin{array}{c}\text { undergraduate } \\
\text { female students: } \\
440(97.7)\end{array}$ & Questionnaire & HPV vaccination & Face-to-face & - & - & - \\
\hline $\begin{array}{l}\text { 8. Maharajan MK et al, } \\
\text { 2015: Malaysia (27) }\end{array}$ & $\begin{array}{l}\text { To assess the } \\
\text { knowledge and de- } \\
\text { termine variation } \\
\text { between different } \\
\text { cultural groups, WTP } \\
\text { for cervical cancer } \\
\text { vaccination and the } \\
\text { relationships between } \\
\text { knowledge and atti- } \\
\text { tudes towards HPV } \\
\text { vaccination }\end{array}$ & $\begin{array}{l}\text { Cross- } \\
\text { Sectional } \\
\text { Study }\end{array}$ & $\begin{array}{l}\text { Ethnically Diverse } \\
\text { Medical Students: } \\
\quad 302 \text { (99) }\end{array}$ & Questionnaires & HPV vaccination & Face-to-face & - & - & - \\
\hline $\begin{array}{l}\text { 9. Kruiroongroj } S \text { et al, } \\
\text { 2014: Thailand (28) }\end{array}$ & $\begin{array}{l}\text { Examine the level of } \\
\text { knowledge, attitude, } \\
\text { acceptance, and } \\
\text { WTP for HPV vac- } \\
\text { cination }\end{array}$ & $\begin{array}{l}\text { Cross- } \\
\text { Sectional } \\
\text { Study }\end{array}$ & $\begin{array}{l}\text { Female parents of } \\
\text { girls aged } 12-15 \\
\text { years: } 861(71.7)\end{array}$ & Questionnaires & HPV vaccination & Face-to-face & $\begin{array}{l}\text { Payment card } \\
\text { technique-open- } \\
\text { ended }\end{array}$ & - & - \\
\hline $\begin{array}{l}\text { 10. Songthap A et al, } \\
\text { 2012: Thailand (29) }\end{array}$ & $\begin{array}{l}\text { Assess the knowledge } \\
\text { and attitudes } \\
\text { about HPV and cervi- } \\
\text { cal cancer, and the } \\
\text { acceptability } \\
\text { of HPV vaccine }\end{array}$ & $\begin{array}{l}\text { Cross- } \\
\text { Sectional } \\
\text { Study }\end{array}$ & $\begin{array}{c}\text { Students: } \\
\text { 644(80.5) } \\
\text { Parents: } 664(83) \\
\text { Teachers: } 304(76)\end{array}$ & Questionnaires & HPV vaccination & Face-to-face & Closed-ended & - & - \\
\hline
\end{tabular}




\begin{tabular}{|c|c|c|c|c|c|c|c|c|c|}
\hline Author, year country & Aim of study & $\begin{array}{l}\text { Study } \\
\text { design }\end{array}$ & $\begin{array}{l}\text { Participants: } \mathrm{N} \\
\text { (response rate) }\end{array}$ & $\begin{array}{l}\text { Data collection } \\
\text { tool }\end{array}$ & $\begin{array}{l}\text { Type of } \\
\text { prevention } \\
\text { methods }\end{array}$ & Administration & $\begin{array}{c}\text { Type of } \\
\text { questions }\end{array}$ & $\begin{array}{l}\text { Informed by .... } \\
\text { information }\end{array}$ & Type of information \\
\hline $\begin{array}{l}\text { 11. Alder S et al, 2015: } \\
\text { Argentina (30) }\end{array}$ & $\begin{array}{c}\text { explore } \\
\text { maternal HPV vaccina- } \\
\text { tion acceptance, WTP for } \\
\text { HPV vaccination and } \\
\text { correlates of this willing- } \\
\text { ness, awareness } \\
\text { of HPV and HPV- } \\
\text { associated disease and } \\
\text { behaviors and attitudes } \\
\text { Associated with HPV } \\
\text { vaccination acceptance. }\end{array}$ & $\begin{array}{l}\text { cross- } \\
\text { sectional } \\
\text { study }\end{array}$ & $\begin{array}{c}\text { mothers of girls } \\
\text { aged } 9-15 \text { year: } \\
180(85.3)\end{array}$ & questionnaires & $\begin{array}{l}\text { HPV vaccina- } \\
\text { tion }\end{array}$ & face-to-face & - & General & $\begin{array}{c}\text { natural history of HPV and } \\
\text { cervical cancer }\end{array}$ \\
\hline $\begin{array}{l}\text { 12. Dinh Thu H et al, } \\
\text { 2018: Vietnam (31) }\end{array}$ & $\begin{array}{l}\text { Identifying mothers' } \\
\text { WTP for HPV vaccine } \\
\text { for daughters, and the } \\
\text { associated factors }\end{array}$ & $\begin{array}{l}\text { Cross- } \\
\text { Sectional } \\
\text { Study }\end{array}$ & $\begin{array}{c}\text { married women } \\
15--49 \text { years old: } \\
606(96)\end{array}$ & Questionnaires & $\begin{array}{l}\mathrm{HPV} \text { vaccina- } \\
\text { tion }\end{array}$ & Face-to-face & $\begin{array}{l}\text { open } \\
\text { ended }\end{array}$ & - & - \\
\hline $\begin{array}{l}\text { 13. Umeh IB et al, } \\
\text { 2016: Nigeria (16) }\end{array}$ & $\begin{array}{l}\text { Assessed Nigerian moth- } \\
\text { ers' WTP for HPV vac- } \\
\text { cine. }\end{array}$ & $\begin{array}{l}\text { Cross- } \\
\text { Sectional } \\
\text { Study }\end{array}$ & $\begin{array}{l}\text { Mothers has girls } \\
\text { aged 9-12: } 438 \\
\text { (88) }\end{array}$ & Questionnaires & $\begin{array}{l}\text { HPV vaccina- } \\
\text { tion }\end{array}$ & Face-to-face & $\begin{array}{l}\text { open } \\
\text { ended- } \\
\text { payment } \\
\text { card tech- } \\
\text { nique }\end{array}$ & - & - \\
\hline $\begin{array}{l}\text { 14. Philips Z et al, 2006: } \\
\text { UK (32) }\end{array}$ & $\begin{array}{l}\text { Experiment to test the } \\
\text { construct validity of } \\
\text { contingent valuation, by } \\
\text { eliciting women's } \\
\text { valuations for the NHS } \\
\text { cervical cancer screening } \\
\text { programme }\end{array}$ & $\begin{array}{c}\text { Randomised } \\
\text { Experiment }\end{array}$ & $\begin{array}{l}\text { women eligible } \\
\text { for cervical } \\
\text { screening during } \\
\text { routine (non- } \\
\text { screening) } \\
\text { consultations: } \\
1524 \text { (25.4) }\end{array}$ & Questionnaires & $\begin{array}{l}\text { Screening ser- } \\
\quad \text { vice }\end{array}$ & Face-to-face & $\begin{array}{l}\text { payment } \\
\text { scale- } \\
\text { (open- } \\
\text { ended) }\end{array}$ & $\begin{array}{l}\text { General and } \\
\text { detailed }\end{array}$ & $\begin{array}{l}\text { Risk factors, incidence, screen- } \\
\text { ing efficacy, HPV testing as } \\
\text { triage for low-grade } \\
\text { abnormal smears and explained } \\
\text { the potential } \\
\text { benefits and uncertainties asso- } \\
\text { ciated with its } \\
\text { Implementation. }\end{array}$ \\
\hline $\begin{array}{l}\text { 15. Yan Yuen WW et } \\
\text { al, 2018: Hong Kong } \\
\text { (33) }\end{array}$ & $\begin{array}{l}\text { Assessing the feasibility } \\
\text { of delivering the HPV } \\
\text { vaccine to girls through a } \\
\text { school-based program in } \\
\text { Hong Kong, as well as to } \\
\text { examine } \\
\text { the facilitators and barri- } \\
\text { ers associated with their } \\
\text { participation }\end{array}$ & $\begin{array}{l}\text { Cross- } \\
\text { Sectional } \\
\text { Study }\end{array}$ & $\begin{array}{c}\text { girls aged } 9 \text { to } \\
\text { 14: } 1147(89.9) \\
\text { parents: } 1160 \\
(90.9)\end{array}$ & Questionnaires & $\begin{array}{l}\text { HPV vaccina- } \\
\text { tion }\end{array}$ & Face-to-face & - & - & - \\
\hline $\begin{array}{l}\text { 16. Liao CH et al, 2009: } \\
\text { Taiwan (34) }\end{array}$ & $\begin{array}{l}\text { Apply the CVM to } \\
\text { elicit the WTP, and } \\
\text { measure the value of a } \\
\text { statistic life } \\
\text { (VSL), for HPV vaccine }\end{array}$ & $\begin{array}{l}\text { Cross- } \\
\text { Sectional } \\
\text { Study }\end{array}$ & $\begin{array}{l}\text { women aged } 20- \\
55 \text { years with at } \\
\text { least one daugh- } \\
\text { ter: } 512\end{array}$ & Questionnaires & $\begin{array}{l}\mathrm{HPV} \text { vaccina- } \\
\text { tion }\end{array}$ & Face-to-face & $\begin{array}{l}\text { double- } \\
\text { bounded } \\
\text { binary- } \\
\text { choice }\end{array}$ & General & - \\
\hline
\end{tabular}




\begin{tabular}{|c|c|c|c|c|c|c|c|c|c|}
\hline Author, year country & Aim of study & $\begin{array}{l}\text { Study } \\
\text { design }\end{array}$ & $\begin{array}{l}\text { Participants: } \mathrm{N} \\
\text { (response rate) }\end{array}$ & $\begin{array}{c}\text { Data collection } \\
\text { tool }\end{array}$ & $\begin{array}{c}\text { Type of } \\
\text { prevention } \\
\text { methods }\end{array}$ & Administration & $\begin{array}{c}\text { Type of } \\
\text { questions }\end{array}$ & $\begin{array}{c}\text { Informed by } \\
\ldots . \\
\text { information }\end{array}$ & Type of information \\
\hline $\begin{array}{l}\text { 17. Raab SS et al, } \\
\text { 2002:USA (35) }\end{array}$ & $\begin{array}{c}\text { WTP for New Papan- } \\
\text { icolaou Test } \\
\text { Technologies and } \\
\text { influence factors }\end{array}$ & $\begin{array}{c}\text { cross- } \\
\text { sectional } \\
\text { study }\end{array}$ & $\begin{array}{l}\text { female patients } \\
\text { who attended } \\
\text { obstetrics- } \\
\text { gynecology } \\
\text { practices: } 175\end{array}$ & questionnaires & $\begin{array}{c}\text { (liquid-based) } \\
\text { Papanicolaou } \\
\text { (Pap) test }\end{array}$ & face-to-face & $\begin{array}{l}\text { payment card } \\
\text { technique }\end{array}$ & detailed & $\begin{array}{l}\text { conventional Pap smear screen- } \\
\text { ing, the, impact of Pap } \\
\text { smear screening, the current } \\
\text { average risk of dying of CC, } \\
\text { new Pap tests and } \\
\text { their potential benefits, charges } \\
\text { for conventional and } \\
\text { liquid-based Pap tests, and the } \\
\text { risks for an average American } \\
\text { of dying of a variety of other } \\
\text { causes (e.g., particular diseases } \\
\text { Accidents, natural disasters). }\end{array}$ \\
\hline $\begin{array}{l}\text { 18. Dahlström LA et } \\
\text { al, 2010: Sweden (36) }\end{array}$ & $\begin{array}{l}\text { Investigated corre- } \\
\text { lates of attitudes to } \\
\text { HPV vaccination }\end{array}$ & $\begin{array}{c}\text { Population- } \\
\text { Based Sur- } \\
\text { vey }\end{array}$ & $\begin{array}{c}\text { Parents of chil- } \\
\text { dren aged } 12-15 \\
\text { years: } \\
\text { Parents of girls: } \\
11187(70 \%) \\
\text { Parents of boys: } \\
2759(69 \%)\end{array}$ & Questionnaires & $\begin{array}{l}\text { HPV vaccina- } \\
\text { tion }\end{array}$ & $\begin{array}{c}\text { Online } \\
\text { face-to-face } \\
\text { Telephone inter- } \\
\text { view }\end{array}$ & - & - & - \\
\hline $\begin{array}{l}\text { 19. Oh JK et al, 2010: } \\
\text { Korea (37) }\end{array}$ & $\begin{array}{l}\text { Awareness and ac- } \\
\text { ceptance of HPV } \\
\text { infection } \\
\text { and vaccination for } \\
\text { CC prevention, as } \\
\text { well as factors asso- } \\
\text { ciated with willing- } \\
\text { ness to be adminis- } \\
\text { tered } \\
\text { the HPV vaccine }\end{array}$ & $\begin{array}{c}\text { Population- } \\
\text { Based Sur- } \\
\text { vey }\end{array}$ & $\begin{array}{l}\text { Male (496) and } \\
\text { female }(504) \\
\text { adults: } 1000 \\
(27.3)\end{array}$ & Questionnaires & $\begin{array}{l}\text { HPV vaccina- } \\
\text { tion }\end{array}$ & Face-to-face & $\begin{array}{c}\text { Closed-ended } \\
\text { questionnaire } \\
\text { including some } \\
\text { multiple } \\
\text { choice question }\end{array}$ & - & - \\
\hline $\begin{array}{l}\text { 20. Rajiah K et al, } \\
\text { 2017: Malaysia (38) }\end{array}$ & $\begin{array}{l}\text { Determine the influ- } \\
\text { ence of dental stu- } \\
\text { dents' knowledge } \\
\text { and attitude } \\
\text { regarding HPV infec- } \\
\text { tion of CC on WTP } \\
\text { for vaccination }\end{array}$ & $\begin{array}{l}\text { Cross- } \\
\text { Sectional } \\
\text { Study }\end{array}$ & $\begin{array}{c}\text { Final } \\
\text { year dental } \\
\text { students from } \\
\text { the School of } \\
\text { Dentistry: } 142 \\
\quad(94.7)\end{array}$ & Questionnaires & $\begin{array}{l}\text { HPV vaccina- } \\
\text { tion }\end{array}$ & Face-to-face & $\begin{array}{l}\text { Opened-ended } \\
\text { questions }\end{array}$ & - & - \\
\hline
\end{tabular}




\begin{tabular}{|c|c|c|c|c|c|c|c|c|c|}
\hline Author, year country & Aim of study & $\begin{array}{r}\text { Study } \\
\text { design }\end{array}$ & $\begin{array}{l}\text { Participants: } \mathrm{N} \\
\text { (response rate) }\end{array}$ & $\begin{array}{c}\text { Data collection } \\
\text { tool }\end{array}$ & $\begin{array}{c}\text { Type of } \\
\text { prevention } \\
\text { methods }\end{array}$ & Administration & $\begin{array}{c}\text { Type of } \\
\text { questions }\end{array}$ & $\begin{array}{c}\text { Informed by } \\
\ldots . . \\
\text { information }\end{array}$ & Type of information \\
\hline $\begin{array}{l}\text { 21. Tran BX et al, 2018: } \\
\text { Vietnam (39) }\end{array}$ & $\begin{array}{l}\text { investigate barriers } \\
\text { related to knowledge- } \\
\text { attitude-practice (KAP) } \\
\text { about the HPV vaccine } \\
\text { and WTP for the vaccine }\end{array}$ & $\begin{array}{c}\text { cross- } \\
\text { sectional } \\
\text { study }\end{array}$ & $\begin{array}{l}\text { vaccination ser- } \\
\text { vice users: } 492\end{array}$ & questionnaires & HPV vaccination & face-to-face & $\begin{array}{c}\text { Double-bounded } \\
\text { dichotomous- } \\
\text { choice questions } \\
\text { with open-ended } \\
\text { questions }\end{array}$ & - & - \\
\hline $\begin{array}{l}\text { 22. Touch S and Oh JK, } \\
\text { 2018: Cambodia (40) }\end{array}$ & $\begin{array}{l}\text { examine the cervical } \\
\text { cancer knowledge, atti- } \\
\text { tudes, and practices as } \\
\text { well as } \\
\text { cervical cancer preven- } \\
\text { tion methods }\end{array}$ & $\begin{array}{l}\text { cross- } \\
\text { sectional } \\
\text { study }\end{array}$ & $\begin{array}{c}\text { women aged } 20- \\
69 \text { years: } \\
440(98.8)\end{array}$ & questionnaires & HPV vaccination & $\begin{array}{l}\text { face-to-face inter- } \\
\text { view survey }\end{array}$ & $\begin{array}{l}\text { close-ended, } \\
\text { multiple-choice } \\
\text { responses and } \\
\text { open-ended }\end{array}$ & - & - \\
\hline $\begin{array}{l}\text { 23. Opoku CA et al, } \\
\text { 2016: Ghana (41) }\end{array}$ & $\begin{array}{l}\text { assessed the perception } \\
\text { of risk of CC and exist- } \\
\text { ence of risk factors for } \\
\text { CC }\end{array}$ & $\begin{array}{l}\text { cross- } \\
\text { sectional } \\
\text { study }\end{array}$ & $\begin{array}{c}\text { women had to be } \\
\text { between the ages } \\
\text { of } \\
18-45 \text { years: } 300 \\
\text { (98.4) }\end{array}$ & $\begin{array}{l}\text { semistructured } \\
\text { questionnaire }\end{array}$ & $\begin{array}{l}\text { screening ser- } \\
\text { vice }\end{array}$ & $\begin{array}{l}\text { face-to-face inter- } \\
\text { view survey }\end{array}$ & - & - & - \\
\hline $\begin{array}{l}\text { 24. Lin Y et al, } \\
\text { 2020:China (42) }\end{array}$ & $\begin{array}{l}\text { investigate acceptance } \\
\text { and willingness to pay } \\
\text { for HPV vaccination } \\
\text { among adult women in } \\
\text { China }\end{array}$ & $\begin{array}{l}\text { cross- } \\
\text { sectional } \\
\text { study }\end{array}$ & $\begin{array}{l}\text { mothers aged } 27- \\
45 \text { years of prima- } \\
\text { ry school pupils: } \\
2339(62)\end{array}$ & questionnaires & HPV vaccines & online & $\begin{array}{l}\text { open ended } \\
\text { single bounded } \\
\text { dichotomous- } \\
\text { choice-open } \\
\text { ended }\end{array}$ & - & - \\
\hline $\begin{array}{l}\text { 25. You D et al, 2020: } \\
\text { China (43) }\end{array}$ & $\begin{array}{l}\text { determine HPV vaccine } \\
\text { uptake and willingness } \\
\text { to receive HPV vaccina- } \\
\text { tion }\end{array}$ & $\begin{array}{l}\text { cross- } \\
\text { sectional } \\
\text { survey }\end{array}$ & $\begin{array}{l}\text { female college } \\
\text { students: } 4220\end{array}$ & questionnaires & HPV vaccines & online & - & - & - \\
\hline
\end{tabular}




\begin{tabular}{|c|c|c|c|c|c|c|c|c|c|}
\hline Author, year country & Aim of study & $\begin{array}{l}\text { Study } \\
\text { design }\end{array}$ & $\begin{array}{l}\text { Participants: } \mathrm{N} \\
\text { (response rate) }\end{array}$ & $\begin{array}{l}\text { Data collection } \\
\text { tool }\end{array}$ & $\begin{array}{l}\text { Type of preven- } \\
\text { tion methods }\end{array}$ & Administration & $\begin{array}{l}\text { Type of ques- } \\
\text { tions }\end{array}$ & $\begin{array}{c}\text { Informed by } \\
\ldots . \\
\text { information }\end{array}$ & Type of information \\
\hline $\begin{array}{l}\text { 26.Lin W et al, 2020: } \\
\text { China (44) }\end{array}$ & $\begin{array}{l}\text { Valuate the differ- } \\
\text { ences on awareness } \\
\text { and attitude towards } \\
\text { HPV and its vaccine } \\
\text { between local and } \\
\text { migrant residents } \\
\text { who participated in } \\
\text { CC screening }\end{array}$ & $\begin{array}{l}\text { Cross- } \\
\text { Sectional } \\
\text { Survey }\end{array}$ & $\begin{array}{l}\text { women aged from } \\
21 \text { to } 60 \text { years: } \\
9855 \text { (93.8) }\end{array}$ & Questionnaires & HPV vaccines & Face-to-face & Open ended & - & - \\
\hline $\begin{array}{l}\text { 27.Kristina } S \text { et al, 2020: } \\
\text { Indonesia (45) }\end{array}$ & $\begin{array}{l}\text { Examine the percep- } \\
\text { tion of seriousness } \\
\text { and knowledge of } \\
\text { CC risk and to eval- } \\
\text { uate the WTP for CC } \\
\text { screening }\end{array}$ & $\begin{array}{c}\text { Community } \\
\text { based cross- } \\
\text { sectional sur- } \\
\text { vey }\end{array}$ & $\begin{array}{l}\text { women who } \\
\text { visited clinics or } \\
\text { pharmacies: } 675\end{array}$ & Questionnaires & $\begin{array}{l}\text { Screening ser- } \\
\text { vice }\end{array}$ & Face-to-face & $\begin{array}{l}\text { Bid contingent } \\
\text { valuation meth- } \\
\text { od }\end{array}$ & - & - \\
\hline $\begin{array}{l}\text { 28.Weng Q et al, 2020: } \\
\text { Tanzania (21) }\end{array}$ & $\begin{array}{l}\text { Describe women's } \\
\text { awareness of CC and } \\
\text { to explore the atti- } \\
\text { tudes toward, ac- } \\
\text { ceptability of and } \\
\text { barriers to CC }\end{array}$ & $\begin{array}{c}\text { Cross- } \\
\text { sectional }\end{array}$ & $\begin{array}{c}\text { women aged } 14- \\
65 \text { years old: } 1483 \\
(98.8)\end{array}$ & Questionnaires & $\begin{array}{l}\text { Screening ser- } \\
\text { vice }\end{array}$ & Face-to-face & $\begin{array}{l}\text { Closed-response } \\
\text { questions }\end{array}$ & - & - \\
\hline
\end{tabular}




\begin{tabular}{|c|c|c|c|c|c|c|c|c|c|}
\hline \multirow[t]{2}{*}{$\begin{array}{l}\text { Author, year } \\
\text { country }\end{array}$} & \multirow[t]{2}{*}{$\begin{array}{l}\text { Demographic } \\
\text { characteristics } \\
\text { of participants }\end{array}$} & \multirow[t]{2}{*}{$\begin{array}{l}\% \text { of ac- } \\
\text { ceptance of } \\
\text { test }\end{array}$} & \multirow[t]{2}{*}{$\begin{array}{l}\text { \% of Posi- } \\
\text { tive WTP }\end{array}$} & \multirow[t]{2}{*}{$\begin{array}{c}\text { WTP (US\$) } \\
\text { M } \pm \text { SD OR medi- } \\
\text { an (CR) }\end{array}$} & \multirow[t]{2}{*}{$\begin{array}{c}\text { WTP as a } \\
\% \text { of } \\
\text { GDP per capita }\end{array}$} & \multirow[t]{2}{*}{ Other results } & \multicolumn{2}{|c|}{$\begin{array}{c}\text { Influenced Variables effects in } \\
\text { WTP } \\
\end{array}$} & \multirow{2}{*}{$\begin{array}{c}\text { Main } \\
\text { reason for } \\
\text { no WTP } \\
(\%)\end{array}$} \\
\hline & & & & & & & Significant positive & non-significant & \\
\hline $\begin{array}{l}\text { 1. Wordsworth S } \\
\text { et al, 2001: Scot- } \\
\text { land (22) }\end{array}$ & $\begin{array}{l}\text { Mean age: } 38 \\
\text { Income range } \\
\text { of } £ 10000 \pm \\
£ 25000 .\end{array}$ & - & 89.3 & $80.8 \pm 51.5$ & 0.49 & $\begin{array}{l}\text { Zero value }=10.7 \% \text { of par- } \\
\text { ticipants }\end{array}$ & $>$ Income & $\begin{array}{c}>\text { Age } \\
>\text { Smear status }\end{array}$ & $>$ \\
\hline $\begin{array}{l}\text { 2. Choi HCW et } \\
\text { al, 2013: Hong } \\
\text { Kong (23) }\end{array}$ & $\begin{array}{c}83 \% \text { of mother } \\
\text { has more than } \\
35 \text { years }\end{array}$ & $\begin{array}{l}\text { Schoolgirls: } \\
27.1 \\
\text { Mothers 2008: } \\
27.5 \\
\text { Mothers 2012: } \\
37.6\end{array}$ & $\begin{array}{l}\text { School- } \\
\text { girls:54.8 } \\
\text { Mothers } \\
\text { 2008:44.6 } \\
\text { Mothers } \\
\text { 2012:66.7 }\end{array}$ & $\begin{array}{l}\text { Schoolgirls: } 38 \\
\quad(13-128) \\
\text { Mothers 2008: } \\
128(77-192) \\
\text { Mothers 2012: } \\
128(64-192)\end{array}$ & $\begin{array}{c}\text { Schoolgirls: } 0.12 \\
\text { Mothers } \\
\text { 2008:0.40 } \\
\text { Mothers 2012: } \\
0.40\end{array}$ & $\begin{array}{l}\text { Perceived minimum age } \\
\text { appropriate for } \\
\text { vaccination (years): } \\
\text { Schoolgirls: } 12 \\
\text { Mothers 2008:15 } \\
\text { Mothers 2012:14 }\end{array}$ & $\begin{array}{c}\text { Mothers: } \\
>\quad \text { Had heard of } \\
\text { HPV vaccines before } \\
>\text { Monthly house- } \\
\text { hold income } \geq 2564 \\
>\quad \text { Age of daugh- } \\
\text { ters: }<9 \text { years } \\
>\quad \text { Perception on the } \\
\text { health of daughters: } \\
\text { Good/Very } \\
\text { good/Excellent } \\
\\
\text { Schoolgirls: } \\
>\text { Had heard of HPV } \\
\text { before } \\
>\text { Risky sexual be- } \\
\text { haviors } \\
>\text { Education attain- } \\
\text { ment } \\
>\text { Age: }>13\end{array}$ & $\begin{array}{cc}\text { Mothers: } \\
>\quad \text { Had heard of HPV } \\
\text { before } \\
>\quad \text { Identified HPV in- } \\
\text { fection as risk factor } \\
>\quad \text { Monthly household } \\
\text { income 1282-2564 } \\
>\quad \text { Education: Second- } \\
\text { ary } \\
>\quad \text { Education: Tertiary } \\
\text { or above } \\
>\quad \text { History of cervical } \\
\text { screening without symp- } \\
\text { toms } \\
\\
>\text { Schoolgirls: } \\
\text { Had heard of HPV } \\
\text { vaccines before } \\
\text { Identified HPV in- } \\
\text { fection as risk factor } \\
>\text { Monthly household } \\
\text { income } \\
>\quad \text { Self-rated health: } \\
\text { Good/Very } \\
\text { good/Excellent }\end{array}$ & \\
\hline $\begin{array}{l}\text { 3. Rajiah K et al, } \\
\text { 2015: Malaysia } \\
\text { (18) }\end{array}$ & mean age: 22.2 & 83.8 & 86 & 108.66 & 0.97 & $\begin{array}{l}\text { - Almost all the students } \\
\text { wanted the vaccine to be } \\
\text { cost free } \\
\text { - Almost half of the re- } \\
\text { spondents were willing to } \\
\text { spend around USD } 200 \text { for } \\
\text { their rhildran }\end{array}$ & - & - & Cost \\
\hline
\end{tabular}




\begin{tabular}{|c|c|c|c|c|c|c|c|c|c|}
\hline Author, year country & $\begin{array}{l}\text { Demographic } \\
\text { characteristics of } \\
\text { participants }\end{array}$ & $\begin{array}{l}\% \text { of acceptance } \\
\text { of test }\end{array}$ & $\%$ of Positive WTP & $\begin{array}{c}\text { WTP (US\$) } \\
\text { M } \pm \text { SD OR medi- } \\
\text { an (CR) }\end{array}$ & $\begin{array}{c}\text { WTP as a } \\
\% \text { of } \\
\text { GDP per capita }\end{array}$ & Other results & \multicolumn{2}{|c|}{$\begin{array}{c}\text { influenced Variables effects in } \\
\text { WTP } \\
\end{array}$} & $\begin{array}{c}\text { Main reason } \\
\text { for no WTP } \\
(\%)\end{array}$ \\
\hline $\begin{array}{l}\text { 4. Tarekegn AA, et } \\
\text { al, 2019: Ethiopia } \\
\text { (24) }\end{array}$ & $\begin{array}{l}\text { mean age: } 28 \\
\text { years } \\
\text { Average monthly } \\
\text { income: US\$ } 226\end{array}$ & - & 83.4 & $7.12 \pm 4.83$ & 0.91 & $\begin{array}{l}34.6 \% \text { of partici- } \\
\text { pants were WTP } \\
\text { more than US } \$ 11\end{array}$ & $\begin{array}{c}\text { significant positive } \\
>\quad \text { Age }>30 y e a r s \\
>\text { perceived seriousness of } \\
\text { cervical cancer } \\
>\text { perceived quality } \\
\text { of cervical screening service } \\
>\text { educational status } \\
>\text { monthly income }\end{array}$ & $\begin{array}{c}\text { non-significant } \\
>\text { Marital status } \\
>\text { Religion } \\
>\text { Ethnicity } \\
>\text { Background profession } \\
>\text { Knowledge } \\
>\text { Health Status } \\
>\text { Source of more Infor- } \\
\quad \text { mation }\end{array}$ & $>$ \\
\hline $\begin{array}{l}\text { 5. Tarekegn AA } \\
\text { and Yismaw AE, } \\
\text { 2019: Ethiopia (25) }\end{array}$ & $\begin{array}{c}\text { mean age: } 28 \\
\text { years } \\
\text { Average monthly } \\
\text { income: US \$226 }\end{array}$ & - & 85.9 & $8.46 \pm 4.83$ & 1.09 & $\begin{array}{l}36.6 \% \text { of partici- } \\
\text { pants were WTP } \\
\text { more than US } \$ 11\end{array}$ & $\begin{array}{c}>\text { Age } \\
>\text { educational status } \\
>\text { Knowledge about cervical } \\
\text { cancer and its risk factors } \\
>\text { monthly income }\end{array}$ & $\begin{array}{c}>\text { Marital status } \\
>\text { Background profession } \\
>\text { Knowledge } \\
>\text { Health status } \\
>\text { Perceived seriousness } \\
\text { cervical cancer }\end{array}$ & $>$ \\
\hline $\begin{array}{l}\text { 6. Philips Z,et al, } \\
\text { 2003: UK (17) }\end{array}$ & $\begin{array}{l}\text { mean age: } 18.9 \\
\text { years } \\
\text { about } 80 \% \\
\text { received annual } \\
\text { incomes of less } \\
\text { than £5000 per } \\
\text { annum, }\end{array}$ & - & - & $35.92 \pm 32.01$ & 0.12 & $\begin{array}{l}\text { WTP for } 10 \% \\
\text { increase in } \\
\text { screening accura- } \\
\text { cy: US } \$ 22.16\end{array}$ & $\begin{array}{c}>\text { WTP for routine smear test } \\
(\mathfrak{E}) \\
>\quad \begin{array}{c}\text { Proportion of smears testing } \\
\text { normal }\end{array} \\
>\quad \begin{array}{c}\text { Perceived above average } \\
\text { risk of cervical cancer }\end{array}\end{array}$ & $\begin{array}{l}>\text { Accuracy of smear test } \\
\text { Age where most abnor- } \\
\text { mal results occur } \\
>\text { Current smoker }\end{array}$ & $>$ \\
\hline $\begin{array}{l}\text { 7. Hoque ME et al, } \\
\text { 2013: South Africa } \\
\text { (26) }\end{array}$ & $\begin{array}{l}\text { mean age: } 20.3 \\
\text { years } \\
63 \% \text { of the } \\
\text { students were } \\
\text { sexually } \\
\text { Experienced. }\end{array}$ & 77.3 & - & - & - & - & $\begin{array}{c}>\quad \text { Age more than } 21 \\
>\text { knew about the Pap smear } \\
\text { test } \\
>\quad \text { were aware that having mul- } \\
\text { tiple sex partners } \\
>\text { sexual intercourse before } \\
\text { the age of } 18 \text { years } \\
>\text { smoking } \\
>\text { having contracted any STDs }\end{array}$ & $>-$ & $>$ \\
\hline $\begin{array}{l}\text { 8. Maharajan MK et } \\
\text { al, 2015: Malaysia } \\
\text { (27) }\end{array}$ & $\begin{array}{l}\text { mean age: } 23.5 \\
\text { years } \\
56.2 \% \text { reported as } \\
\text { being in a rela- } \\
\text { tionship }\end{array}$ & 89.7 & 87.75 & 152.48 & 1.34 & $\begin{array}{l}30 \% \text { affirmed that } \\
\text { they could } \\
\text { not afford the total } \\
\text { cost of the three } \\
\text { doses of HPV } \\
\text { vaccine }\end{array}$ & $>-$ & $>-$ & $>\operatorname{cost}$ \\
\hline $\begin{array}{l}\text { 9. Kruiroongroj S et } \\
\text { al, 2014: Thailand } \\
\text { (28) }\end{array}$ & $\begin{array}{l}\text { mean age: } \\
\text { 43.47years } \\
\text { Monthly house- } \\
\text { hold income: } \\
\text { about } 33 \% \text { in } \\
3.300 \text { to } 10000 \\
\text { US\$ }\end{array}$ & $\begin{array}{l}\text { Bivalent: } 76.9 \\
\text { Quadrivalent: } \\
\quad 74.4\end{array}$ & $\begin{array}{c}\text { Bivalent: } 68.9 \\
\text { Quadrivalent: } 67.3\end{array}$ & $\begin{array}{l}\text { Bivalent: } 24.5 \\
\text { (16.3-32.7) } \\
\text { Quadrivalent: } \\
32.7(16.3-49)\end{array}$ & $\begin{array}{c}\text { Bivalent } 0.40 \\
\text { Quadrivalent: } 0.53\end{array}$ & $\begin{array}{l}\text { Participants would } \\
\text { pay more for } \\
\text { quadrivalent } \\
\text { vaccine as com- } \\
\text { pared to bivalent } \\
\text { vaccine. }\end{array}$ & $>\quad-$ & $>-$ & $\begin{array}{c}\text { financial } \\
\text { limitations } \\
(39-43)\end{array}$ \\
\hline
\end{tabular}

$12 \quad$ http://mjiri.iums.ac.ir 


\begin{tabular}{|c|c|c|c|c|c|c|c|c|c|}
\hline Author, year country & $\begin{array}{c}\text { Demographic } \\
\text { characteristics of } \\
\text { participants }\end{array}$ & $\begin{array}{l}\text { \% of acceptance } \\
\text { of test }\end{array}$ & $\%$ of Positive WTP & $\begin{array}{c}\text { WTP (US\$) } \\
\text { M } \pm \text { SD OR median } \\
\text { (CR) }\end{array}$ & $\begin{array}{c}\text { WTP as a } \\
\% \text { of } \\
\text { GDP per capita }\end{array}$ & Other results & \multicolumn{2}{|c|}{$\begin{array}{c}\text { influenced Variables effects in } \\
\text { WTP } \\
\end{array}$} & $\begin{array}{c}\text { Main reason for no WT } \\
(\%)\end{array}$ \\
\hline $\begin{array}{l}\text { 10. Songthap A et } \\
\text { al, 2012: Thailand } \\
\text { (29) }\end{array}$ & $\begin{array}{c}\text { Students mean } \\
\text { age: } 13.1 \\
\text { Parents: mean } \\
\text { age: } 43.2 \\
\text { mean monthly } \\
\text { income of USD } \\
1,116.40 \\
\text { Teachers mean } \\
\text { age: } 46.1 \\
\text { mean monthly } \\
\text { income was USD } \\
1,787.50\end{array}$ & $\begin{array}{l}\text { Students: } 26.1 \\
\text { Parents: } 44.8 \\
\text { Teachers: } 43\end{array}$ & - & $\begin{array}{c}\text { Students: } \\
<14.2: 12.9 \% \\
14.3-57.1: 56.1 \% \\
57.2-114.3: 31 \% \\
\text { Parents: } \\
<14.2: 16.9 \% \\
\text { 14.3-57.1: } 71.1 \% \\
57.2-114.3: 12 \% \\
\text { Teachers: } \\
<14.2: 27.3 \% \\
\text { 14.3-57.1: } 59.1 \% \\
\text { 57.2-114.3: } 13.6 \%\end{array}$ & 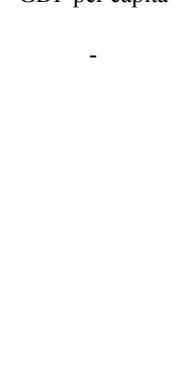 & - & $\begin{array}{c}\text { significant positive } \\
\supset \quad-\end{array}$ & $\underset{\text { non-significant }}{>}$ & - \\
\hline $\begin{array}{l}\text { 11. Alder S et al, } \\
\text { 2015: Argentina } \\
\text { (30) }\end{array}$ & median age: 37 & 90.1 & 59.8 & $30.28(0.91-165.8)$ & 0.23 & $\begin{array}{l}\text { About } 12 \% \\
\text { were willing to } \\
\text { vaccinate their } \\
\text { daughter re-- } \\
\text { gardless of the } \\
\text { cost }\end{array}$ & $\begin{array}{c}>\text { having a high } \\
\text { school education or } \\
\text { more } \\
>\quad \text { gainful em- } \\
\text { ployment } \\
\text { a disposable } \\
\text { household income } \\
\text { of } 438-1,050 \text { eu- } \\
\text { ro/month } \\
\text { being aware of } \\
\text { cervical cancer prior } \\
\text { to the study }\end{array}$ & 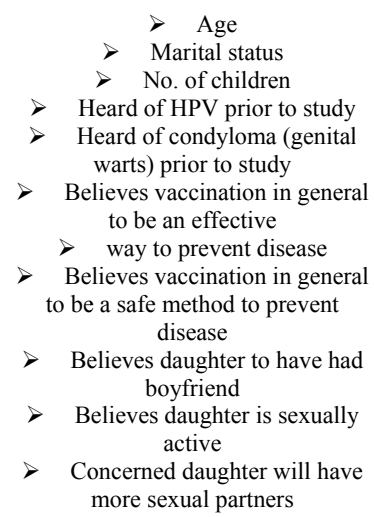 & - \\
\hline $\begin{array}{l}\text { 12. Dinh Thu H et } \\
\text { al, 2018: Vietnam } \\
\text { (31) }\end{array}$ & $\begin{array}{l}\text { About } 70 \% \text { has } \\
36-49 \text { years }\end{array}$ & - & 53.1 & $34.5(23-46)$ & 1.47 & $\begin{array}{l}65.6 \% \text { viewed } \\
\text { the cost as } \\
\text { Expensive or } \\
\text { Very Expensive }\end{array}$ & $\begin{array}{c}>\text { perceived the cost } \\
\text { as acceptable } \\
>\text { better knowledge } \\
\text { on HPV } \\
>\text { Not being a } \\
\text { farmer } \\
>\text { Previously } \\
\text { screened for cervi- } \\
\text { cal cancer }\end{array}$ & $\begin{array}{c}>\text { Age } \\
>\text { Not Kinh people } \\
>\text { Urban commune } \\
>\text { Higher education } \\
>\text { Having } 1--2 \text { children } \\
>\text { Poor household } \\
>\text { Positive attitude } \\
>\text { always use condom }\end{array}$ & $\begin{array}{c}>\text { No information on } \\
\text { HPV / HPV vaccine } \\
\quad(60-67 \%) \\
>\text { living far from } \\
\text { health facilities } \\
>\quad(12.6-13.8 \%), \\
>\text { High cost }(9.2- \\
\quad 19.7 \%) \\
>\quad \text { Not Considering } \\
\text { vaccination as im- } \\
\text { portant }(4 \%) \\
>\text { Afraid that the vac- } \\
\text { cine was unsafe }(4 \%) .\end{array}$ \\
\hline
\end{tabular}




\begin{tabular}{|c|c|c|c|c|c|c|c|c|c|}
\hline Author, year country & $\begin{array}{c}\text { Demographic } \\
\text { characteristics of } \\
\text { participants }\end{array}$ & $\begin{array}{l}\% \text { of acceptance } \\
\text { of test }\end{array}$ & $\%$ of Positive WTP & $\begin{array}{l}\mathrm{WTP} \text { (US\$) M } \pm \text { SD OR } \\
\text { median (CR) }\end{array}$ & $\begin{array}{c}\text { WTP as a } \\
\% \text { of } \\
\text { GDP per capita }\end{array}$ & Other results & \multicolumn{2}{|c|}{$\begin{array}{c}\text { influenced Variables effects in } \\
\text { WTP }\end{array}$} & $\begin{array}{c}\text { Main reason } \\
\text { for no WTP } \\
(\%)\end{array}$ \\
\hline $\begin{array}{l}\text { 13.Umeh IB et al, } \\
\text { 2016: Nigeria (16) }\end{array}$ & $\begin{array}{c}\text { About } 75 \% \text { has } \\
31--50 \text { years. } \\
57.6 \% \text { : house- } \\
\text { hold monthly } \\
\text { income } \\
\text { less than }<\text { US\$ } \\
251\end{array}$ & 92.5 & 91.6 & 11.6 & 0.43 & $\begin{array}{l}\text { most fre- } \\
\text { quently stated } \\
\text { amount was } \\
\text { US\$ } 5.02\end{array}$ & $\begin{array}{c}\text { significant positive } \\
>\text { mothers living in an rural } \\
>\text { previously diagnosed of HPV } \\
\text { infection }\end{array}$ & $\underset{>}{\substack{\text { non-significant } \\
>\text { - }}}$ & $>-$ \\
\hline $\begin{array}{l}\text { 14.Philips } Z \text { et al, } \\
\text { 2006: UK (32) }\end{array}$ & $\begin{array}{l}\text { About } 53 \% \text { has } \\
\text { more than } 40 \\
\text { years. }\end{array}$ & - & 79.8 & 282.32 & 0.63 & $\begin{array}{l}\text { About } 25 \% \\
\text { WTP more } \\
\text { than US\$344 }\end{array}$ & $>-$ & $>-$ & $>-$ \\
\hline $\begin{array}{l}\text { 15.Yan Yuen WW } \\
\text { et al, 2018: Hong } \\
\text { Kong (33) }\end{array}$ & - & $\begin{array}{l}\text { girls: } 84.9 \\
\text { Parents: } 87.1\end{array}$ & - & $64.5 \%$ of participants: 125 & 0.29 & $\begin{array}{c}\text { About } 8 \% \\
\text { WTP more } \\
\text { than US\$125 }\end{array}$ & $\begin{array}{c}\text { Parents: } \\
>\text { heard of the HPV vaccine } \\
>\text { Having correct knowledge of } \\
\text { CC } \\
>\text { knowledge that the HPV } \\
\text { vaccine does not affect growth } \\
>\text { perception that the vaccine } \\
\text { could protect their daughter } \\
>\text { doctor recommended the } \\
\text { vaccine } \\
>\text { not had a regular family doctor } \\
>\text { preference for their daughter } \\
\text { to receive the vaccine at school }\end{array}$ & & $\begin{array}{l}>\quad \text { fear } \\
\text { of side } \\
\text { effects } \\
\text { (52) } \\
>\text { not } \\
\text { think the } \\
\text { vaccine } \\
\text { was } \\
\text { effective } \\
\quad(46) \\
>\quad \text { per- } \\
\text { ception of } \\
\text { promiscu- } \\
\text { ity (2) }\end{array}$ \\
\hline $\begin{array}{l}\text { 16.Liao CH et al, } \\
\text { 2009: Taiwan (34) }\end{array}$ & - & - & - & $\begin{array}{l}\text { US\$1098 to US\$1233 } \\
\text { (US\$913-1004) }\end{array}$ & $\begin{array}{l}6.06 \text { to } 6.81 \\
(5.04-5.54)\end{array}$ & $\begin{array}{l}\text { VSL was } \\
\text { estimated at } \\
\text { approximate- } \\
\text { ly US\$0.65 to } \\
\text { US\$4.09 } \\
\text { (US\$0.56- } \\
3.16) \text { million }\end{array}$ & - & $>$ & \\
\hline $\begin{array}{l}\text { 17.Raab SS et al, } \\
\text { 2002:USA (35) }\end{array}$ & $\begin{array}{c}\text { Mean age: } 39 \\
\text { About } 33 \% \text { has } \\
\text { more than } 50.000 \\
\$ \text { annual house- } \\
\text { hold income }\end{array}$ & - & - & $\begin{array}{l}\text { reduced the risk of dying of } \\
\text { CC from } 1 \text { in } 37,000 \text { to } 1 \text { in } \\
50,000: \\
237\end{array}$ & 0.65 & $\begin{array}{c}\text { No statistical- } \\
\text { ly significant } \\
\text { differences } \\
\text { were seen in } \\
\text { the } \\
\text { mean WTP at } \\
\text { different new } \\
\text { Pap test } \\
\text { performance } \\
\text { Levels. }\end{array}$ & $\begin{array}{c}>\text { more than } 2 \text { children } \\
>\text { Highest education level } \\
>\text { Marital status } \\
>\text { Age } \\
>\text { Perception of high risk for } \\
\text { cervical cancer }\end{array}$ & $>$ & $>$ \\
\hline
\end{tabular}

$14 \quad$ http://mjiri.iums.ac.ir 


\begin{tabular}{|c|c|c|c|c|c|c|c|c|c|}
\hline Author, year country & $\begin{array}{c}\text { Demographic } \\
\text { characteristics of } \\
\text { participants }\end{array}$ & $\begin{array}{c}\% \text { of acceptance } \\
\text { of test }\end{array}$ & $\%$ of Positive WTP & $\begin{array}{l}\text { WTP (US\$) M } \pm \text { SD OR } \\
\text { median (CR) }\end{array}$ & $\begin{array}{l}\text { WTP as a } \\
\% \text { of } \\
\text { GDP per }\end{array}$ & Other results & \multicolumn{2}{|c|}{$\begin{array}{c}\text { influenced Variables effects in } \\
\text { WTP }\end{array}$} & $\begin{array}{l}\text { Main reason for no } \\
\text { WTP (\%) }\end{array}$ \\
\hline $\begin{array}{l}\text { 18. Touch S and Oh } \\
\text { JK, 2018: Cambodia } \\
\text { (40) }\end{array}$ & $\begin{array}{l}60 \% \text { has more } \\
\text { than } 40 \text { years. } \\
\text { About } 44 \% \text { has } \\
\text { Low (US\$ 0- } \\
124 \text { ) family } \\
\text { income (monthly) }\end{array}$ & 62 & 35.6 & $20.5 \pm 8.1$ & $\begin{array}{c}\text { capita } \\
1.61\end{array}$ & $\begin{array}{l}62 \% \text { Willing- } \\
\text { ness to vac- } \\
\text { cinate their } \\
\text { daughter } \\
\text { against HPV }\end{array}$ & 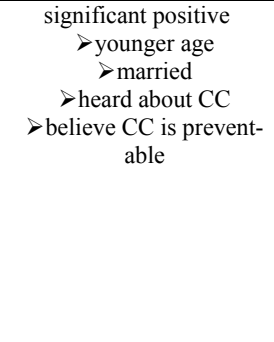 & $\begin{array}{ll} & \text { non-significant } \\
>\text { high Education } \\
>\text { family income } \\
>\text { Number of Children }\end{array}$ & $\begin{array}{c}>\text { High cost (32.7) } \\
>\text { Lack of } \\
\text { knowledge (25) } \\
>\text { Don't know } \\
\text { where to get HPV } \\
\text { vaccine (4.5) } \\
>\text { Don't trust vac- } \\
\text { cine safety (5.2) } \\
>\text { No risk as not } \\
\text { exposed to sexual } \\
\text { contact (3.5) }\end{array}$ \\
\hline $\begin{array}{l}\text { 19. Opoku CA et al, } \\
\text { 2016: Ghana (41) }\end{array}$ & $\begin{array}{l}\text { mean age: } 28 \\
27 \% \text { were } \\
\text { in a polygamous } \\
\text { relationship }\end{array}$ & 97 & 76 & - & - & - & $>-$ & $>-$ & $>-$ \\
\hline $\begin{array}{l}\text { 20.Lin Y et al, } \\
\text { 2020:China(42) }\end{array}$ & $\begin{array}{c}\text { majority of the } \\
\text { respondents were } \\
\text { age 31-35 years } \\
\text { annual household } \\
\text { income of about } \\
7-17\end{array}$ & 58.5 & $\begin{array}{c}2 \mathrm{vHPV} \\
(81.2) 4 \mathrm{vHPV}(75.9) \\
9 \mathrm{vHPV}(67.7) \\
\text { Mean:74.9 }\end{array}$ & - & - & - & $\begin{array}{c}>\text { Household income } \\
>\text { mass media exposure to } \\
\text { HPV vaccination } \\
>\text { perceived self-efficacy } \\
\text { in HPV vaccination } \\
>\text { spouse/partner approval } \\
>\text { Single mothers and } \\
\text { mothers who were di- } \\
\text { vorced, separated or wid- } \\
\text { owed } \\
>\end{array}$ & $\begin{array}{c}>\text { Age } \\
>\text { Ethnicity } \\
>\text { Place of birth } \\
>\text { Highest education level } \\
>\text { Occupation type } \\
>\text { Experience with cervical } \\
>\text { cancer } \\
>\text { HPV knowledge } \\
>\text { Health belief model } \\
>\text { Perceived severity } \\
>\text { Perceived benefit } \\
>\text { Perceived barriers }\end{array}$ & $>-$ \\
\hline
\end{tabular}




\begin{tabular}{|c|c|c|c|c|c|c|c|c|c|}
\hline Author, year country & $\begin{array}{c}\text { Demographic } \\
\text { characteristics of } \\
\text { participants }\end{array}$ & $\begin{array}{l}\% \text { of acceptance } \\
\text { of test }\end{array}$ & $\%$ of Positive WTP & $\begin{array}{c}\text { WTP (US\$) } \\
\mathrm{M} \pm \text { SD OR } \\
\text { median (CR) }\end{array}$ & $\begin{array}{c}\text { WTP as a } \\
\% \text { of } \\
\text { GDP per capita }\end{array}$ & Other results & \multicolumn{2}{|c|}{$\begin{array}{c}\text { influenced Variables effects in } \\
\text { WTP } \\
\end{array}$} & $\begin{array}{c}\text { Main reason } \\
\text { for no WTP } \\
(\%)\end{array}$ \\
\hline $\begin{array}{l}\text { 21.You D et al,2020: } \\
\text { China(43) }\end{array}$ & $\begin{array}{l}\text { majority of the } \\
\text { respondents were } \\
\text { age } 19-22\end{array}$ & 53.5 & - & 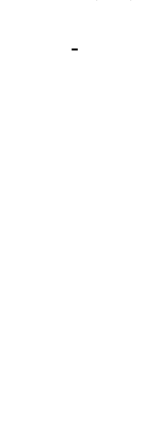 & 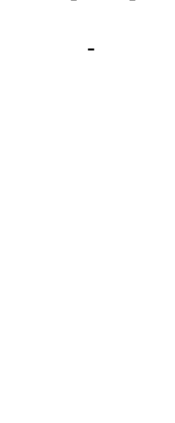 & - & $\begin{array}{c}\text { significant positive } \\
>\text { Age group } \\
>\text { Birthplace } \\
>\text { Location of school } \\
>\text { Central China } \\
>\text { Year of study } \\
>\text { Maternal educational level } \\
>\text { Paternal educational level } \\
>\text { Monthly disposable fund } \\
>\text { Perceived family economic status } \\
>\text { Sexual risk profile } \\
>\text { HPV knowledge } \\
>\text { Attitudes } \\
>\text { Perceived benefit } \\
>\text { Perceived barriers }\end{array}$ & $\begin{array}{c}\text { non-significant } \\
>\quad \text { Ethnicity } \\
>\text { Study program } \\
>\text { Perceived severity }\end{array}$ & $>$ \\
\hline $\begin{array}{l}\text { 22.Lin W et al, } \\
\text { 2020: China(44) }\end{array}$ & $\begin{array}{l}\text { mean age was } \\
37.09 \text { years } \\
\text { The majority of } \\
\text { them were mar- } \\
\text { ried }(90.6 \%)\end{array}$ & 63.3 & 30 & - & - & $\begin{array}{c}\text { Local residents had } \\
\text { a relatively higher } \\
\text { awareness of HPV } \\
\text { and its vaccine, as } \\
\text { well as a higher } \\
\text { willingness to re- } \\
\text { ceive HPV vaccina- } \\
\text { tion than non- } \\
\text { permanent residents } \\
\text { and floating popula- } \\
\text { tion. }\end{array}$ & $\begin{array}{c}>\quad \text { younger ages } \\
>\quad \text { being local residents } \\
>\text { higher levels of education } \\
>\quad \text { being married } \\
>\text { high monthly income } \\
>\text { having daughter(s) } \\
>\text { heard of HPV } \\
>\text { heard of HPV vaccine }\end{array}$ & $\begin{array}{c}>\text { Race } \\
>\text { Medical insurance } \\
>\text { Age at menarche } \\
>\text { Age at sex debut } \\
>\text { No. of the sexual part- } \\
\text { ners in the past } 6 \text { month }\end{array}$ & $>-$ \\
\hline $\begin{array}{l}\text { 23. Kristina } S \text { et al, } \\
\text { 2020: Indonesia(45) }\end{array}$ & $\begin{array}{l}\text { majority of the } \\
\text { respondent } \\
(31.5 \%) \text { had } 46 \\
\text { and more age year }\end{array}$ & - & 67.1 & $3.94 \pm 1.64$ & 0.1 & - & $\begin{aligned} & >\text { Age } \\
& >\quad \text { Monthly income } \\
> & \text { Family history of cancer } \\
> & \text { Private insurance status } \\
& >\quad \text { Knowledge } \\
> & \text { Pérception on cancer risk }\end{aligned}$ & $\begin{array}{c}>\text { Education } \\
>\text { Marital status } \\
>\text { Perceived health status } \\
>\text { Perceived quality of } \\
\text { service } \\
>\text { Source of information } \\
>\text { Experience in Pap } \\
\text { smear test }\end{array}$ & $>$ \\
\hline $\begin{array}{l}\text { 24. Weng Q et al, } \\
\text { 2020: Tanzania (21) }\end{array}$ & $\begin{array}{l}\text { the mean age was } \\
32.86 \text { years }\end{array}$ & 87.9 & 57.4 & - & - & $\begin{array}{l}\text { Only } 4.38 \% \text { of the } \\
\text { respondents had } \\
\text { previously received } \\
\text { CC screening }\end{array}$ & $\begin{aligned} &>\text { Age } \\
&> \text { Marital States } \\
&>\text { Parity } \\
&> \text { Education level } \\
&>\text { Family Income } \\
&>\text { Disease History } \\
&>\text { Family Cancer History }\end{aligned}$ & $\begin{array}{c}>\text { Ethnicity } \\
>\text { First Sex Age } \\
>\text { Genetic Disease }\end{array}$ & $>$ \\
\hline
\end{tabular}

$16 \quad$ http://mjiri.iums.ac.ir 


\begin{tabular}{|c|c|c|c|c|c|c|c|c|c|}
\hline Author, year country & $\begin{array}{c}\text { Demographic } \\
\text { characteristics of } \\
\text { participants }\end{array}$ & $\begin{array}{l}\% \text { of acceptance } \\
\text { of test }\end{array}$ & $\begin{array}{l}\% \text { of } \\
\text { Positive } \\
\text { WTP }\end{array}$ & $\begin{array}{l}\text { WTP (US\$) M } \pm \text { SD } \\
\text { OR median (CR) }\end{array}$ & $\begin{array}{c}\text { WTP as a } \\
\% \text { of } \\
\text { GDP per capita }\end{array}$ & Other results & \multicolumn{2}{|c|}{$\begin{array}{c}\text { influenced Variables effects in } \\
\text { WTP }\end{array}$} & $\begin{array}{l}\text { Main reason for no } \\
\text { WTP (\%) }\end{array}$ \\
\hline $\begin{array}{l}\text { 25.Dahlström LA et } \\
\text { al,2010: Sweden } \\
\text { (36) }\end{array}$ & $\begin{array}{c}\text { mean age: } 44 \\
\text { about } 70 \% \text { of the } \\
\text { participants lived } \\
\text { in rural }\end{array}$ & 76 & 63 & - & 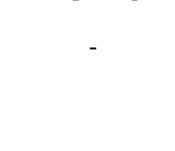 & - & $\begin{array}{c}\text { significant positive } \\
>\text { Female gender of child } \\
>\text { believes vaccines are safe } \\
>\text { Believes vaccines are efficient }\end{array}$ & $\begin{array}{c}\text { non-significant } \\
>\text { Gender of par- } \\
\text { ent } \\
>\text { Believes child } \\
\text { has had coition }\end{array}$ & - \\
\hline & & & & & & & $\begin{array}{c}>\text { Age } \\
>\text { Education } \\
>\text { Employment } \\
>\text { family income } \\
>\text { martial situation } \\
>\text { has 2 Number of children } \\
>\text { living in rural } \\
>\text { Have heard about HPV }\end{array}$ & & \\
\hline & & & & & & & $\begin{array}{l}>\text { Worried child will have more } \\
\text { partners } \\
>\text { Believes child has had girl- } \\
\text { friend/boy friend }\end{array}$ & & \\
\hline $\begin{array}{l}\text { 26.Oh JK et al, } \\
\text { 2010: Korea (37) }\end{array}$ & $\begin{array}{l}\text { About } 56 \% \text { has } \\
\text { more than } 40 \\
\text { years. } \\
\text { About } 56 \% \text { has } \\
\text { middle (2000- } \\
4000 \text { USD) } \\
\text { income per } \\
\text { month }\end{array}$ & $\begin{array}{l}\text { men } \\
\text { and women:55 } \\
\text { participants' } \\
\text { daughters: } 77\end{array}$ & - & - & - & $\begin{array}{c}35.5 \% \text { of men and } \\
39.1 \% \text { of } \\
\text { women suggested } \\
\text { under US\$ } 50\end{array}$ & $\begin{array}{c}>\quad \text { Aged under than } 50 \text { years } \\
>\quad \text { education } \\
>\text { income }\end{array}$ & $>\quad \begin{array}{c}\text { Sex } \\
\text { Living in a } \\
\text { small town }\end{array}$ & $>$ \\
\hline $\begin{array}{l}\text { 27.Rajiah K et al, } \\
\text { 2017: Malaysia (38) }\end{array}$ & $\begin{array}{l}66.2 \% \text { of stu- } \\
\text { dents were } \\
\text { female } \\
78.9 \% \text { of } \\
\text { The respondents } \\
\text { were in a rela- } \\
\text { tionship. }\end{array}$ & - & - & 397.6 & 3.56 & $\begin{array}{l}\text { Students were } \\
\text { WTP US\$ } \\
\text { 450.6vaccinate } \\
\text { their } \\
\text { children in the } \\
\text { future }\end{array}$ & $>$ More knowledge towards CC & $\begin{array}{l}>\text { attitudes to- } \\
\text { wards vaccines }\end{array}$ & $>$ \\
\hline $\begin{array}{l}\text { 28.Tran BX et al, } \\
\text { 2018: Vietnam(39) }\end{array}$ & $\begin{array}{c}\text { mean age was } \\
26.8 \\
\text { average monthly } \\
\text { household in- } \\
\text { come was US\$ } \\
667\end{array}$ & - & 86.6 & $49.3(44.4-54.3)$ & 2.27 & $\begin{array}{c}\text { Male WTP is } \\
\text { more than Female }\end{array}$ & $\begin{array}{c}>\text { Age } 20-29 \text { years } \\
>\text { High household income } \\
>\text { education } \\
>\text { Has children }>6 \text { years old } \\
>\text { Adult male } \\
>\text { Believes that HPV vaccine is } \\
\text { effective } \\
>\text { Has ever examined reproductive } \\
\text { health } \\
>\text { informed about HPV by except } \\
\text { doctors, nurses, or other health pro- } \\
\text { fessionals }\end{array}$ & $\begin{array}{l}>\text { Has family } \\
\text { member who ever } \\
\text { had sexually } \\
\text { transmitted infec- } \\
\text { tion }\end{array}$ & $\begin{array}{c}7 \text { being male } \\
\text { (33.3), high cost } \\
\text { (38.2), the vac- } \\
\text { cine being seen } \\
\text { as unnecessary } \\
\text { (34.5) }\end{array}$ \\
\hline
\end{tabular}


Amounts of Money (in US dollars) for Willingness to Pay

Out of the 28 articles included in the study, 11 studies (14 data) were meta-analysis of monetary amounts (in US\$) that individuals were willing to pay for CCP methods. The results showed that the average money that peo- ple are willing to pay is US\$ 30.44 (95\% CI; 25.6-35.2) (Fig. 4).

The results of the heterogeneous assessment also showed that the results of the studies are highly heterogeneous $\left(\mathrm{Q}=3888.3 ; \mathrm{df}=13 ; \mathrm{I}^{2}=99.6 ; \mathrm{p}<0.001\right)$. $\underline{\text { Study name }}$

$\begin{array}{lrrrr} & \begin{array}{c}\text { Event } \\ \text { rate }\end{array} & \begin{array}{c}\text { Lower } \\ \text { limit }\end{array} & \begin{array}{c}\text { Upper } \\ \text { limit }\end{array} & \text { Z-Value } \\ \text { Wordsworth S et al, 2001 } & 0.089 & 0.069 & 0.115 & 16.154- \\ \text { Choi HCW et al, 2013 (1) } & 0.045 & 0.034 & 0.059 & 20.222- \\ \text { Choi HCW et al, 2013 (2) } & 0.067 & 0.053 & 0.084 & 20.870- \\ \text { Choi HCW et al, 2013 (3) } & 0.055 & 0.046 & 0.065 & 30.756- \\ \text { Rajiah K et al, 2015 } & 0.086 & 0.058 & 0.126 & 10.949- \\ \text { Tarekegn AA, et al, 2019 } & 0.083 & 0.060 & 0.115 & 13.122- \\ \text { Tarekegn AA and Yismaw AE, 20190.086 } & 0.062 & 0.118 & 13.120- \\ \text { Maharajan MK et al, 2015 } & 0.088 & 0.061 & 0.125 & 11.512- \\ \text { Kruiroongroj S et al, 2014 (1) } & 0.069 & 0.054 & 0.088 & 19.351- \\ \text { Kruiroongroj S et al, 2014 (2) } & 0.067 & 0.052 & 0.086 & 19.327- \\ \text { Alder S et al, 2015 } & 0.060 & 0.033 & 0.105 & 8.765- \\ \text { Dinh Thu H et al, 2018 } & 0.053 & 0.038 & 0.074 & 15.903- \\ \text { Umeh IB et al, 2016 } & 0.092 & 0.068 & 0.122 & 13.850- \\ \text { Philips Z et al, 2006 } & 0.080 & 0.067 & 0.095 & 25.866- \\ \text { Dahlström LA et al,2010 } & 0.063 & 0.059 & 0.067 & 77.456- \\ \text { Tran BX et al, 2018 } & 0.087 & 0.065 & 0.115 & 14.697- \\ \text { Touch S and Oh JK, 2018 } & 0.036 & 0.022 & 0.058 & 12.823- \\ \text { Opoku CA et al, 2016 } & 0.076 & 0.051 & 0.112 & 11.465- \\ \text { Lin Y et al, 2020 } & 0.075 & 0.065 & 0.086 & 32.002- \\ \text { Kristina S et al, 2020 } & 0.067 & 0.051 & 0.089 & 17.109- \\ \text { Lin W et al, 2020 } & 0.030 & 0.027 & 0.034 & 58.866- \\ \text { Weng Q et al, 2020 } & 0.057 & 0.047 & 0.070 & 25.069- \\ & 0.066 & 0.057 & 0.076 & 32.978-\end{array}$

\section{Event rate and $95 \%$ CI}

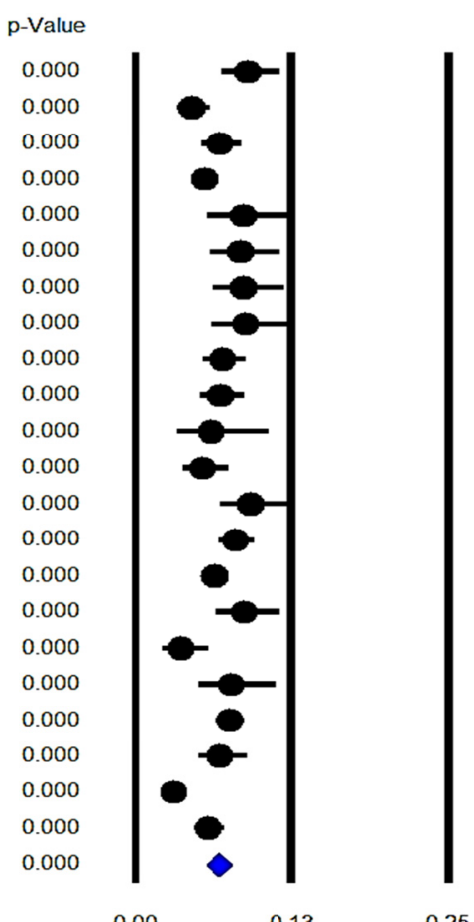

Fig. 3. Meta-analysis of the Positive WTP Rate of Cervical Cancer Prevention Methods Based on a Random Effect Model With 95\% Confidence Level

Study name

Choi HCW et al, 2013 (1)
Choi HCW et al, 2013 (2)
Choi HCW et al, 2013 (3)
Kruiroongroj Set al, 2014 (1)
Kruiroongroj S et al, 2014 (2)
Alder S et al, 2015
Dinh Thu H et al, 2018
Tran BX et al, 2018
Wordsworth S et al, 2001
Tarekegn AA, et al, 2019
Terekegn AA and Yismaw AE, 2019
Philips Z,et al, 2003
Touch S and Oh JK, 2018
Kristina S et al, 2020

Statistics for each study

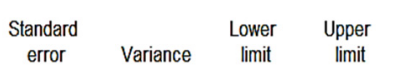

$$
\text { Mean }
$$

128.000

128.000

38.000

24.500

32.700

32.280

34.500

49.300

80.800

7.120

8.460

35.900

20.500

3.940

30.442

\subsection{0}

32614

32.614
12748

12.748

4.178

8.330

15.90

5.856

2.519

2.113

0.242

0.254

2.157

0.407

0.064

2.445 $\begin{array}{lll}675.482 & 77.060 \quad 178.940\end{array}$

$1063.692 \quad 64.077 \quad 191.923$

$\begin{array}{lll}162.524 & 13.013 \quad 62.987\end{array}$

$17.455 \quad 16.312$

$69.393 \quad 16.373$

$252.882 \quad 1.112$

$34.289 \quad 23.023$

$6.347 \quad 44.362$

$4.465 \quad 76.658$

$0.058 \quad 6.646$

$0.065 \quad 7.962$

$4.651 \quad 31.673$

$0.166 \quad 19.702$

$0.004 \quad 3.815$

$5.977 \quad 25.650$

32.688

32.688

49.027

63.448

45.977

$54.238 \quad 19.569$

$84.942 \quad 38.238$

$7.594 \quad 29.470$

$8.958 \quad 33.266$

$40.127 \quad 16.647$

$21.298 \quad 50.363$

$4.065 \quad 61.889$

$35.234 \quad 12.451$

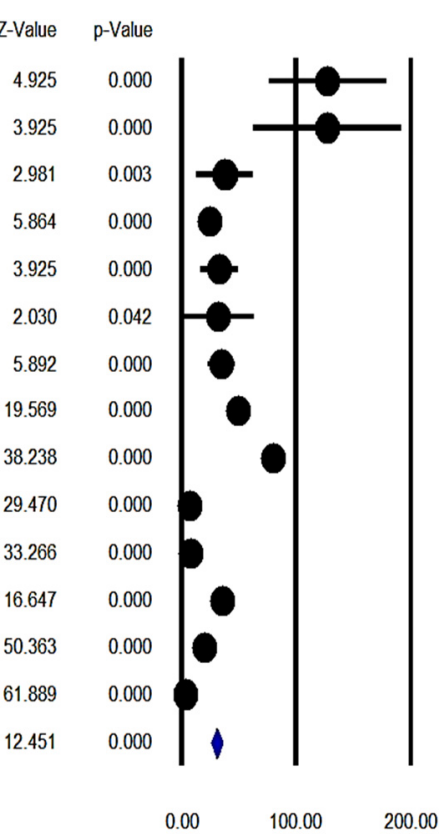

Fig. 4. Meta-analysis Results (in US\$) of Individuals' Willingness to Pay for Cervical Cancer Prevention Methods Based on a Random Effect Model with 95\% Confidence Level 
Study name

$\begin{array}{lr}\text { Wordsworth S et al, 2001 } & \begin{array}{c}\text { Event } \\ \text { rate }\end{array} \\ \text { Choi HCW et al, 2013 (1) } & 0.0005 \\ \text { Choi HCW et al, 2013 (2) } & 0.0004 \\ \text { Choi HCW et al, 2013 (3) } & 0.0004 \\ \text { Rajiah K et al, 2015 } & 0.0001 \\ \text { Tarekegn AA, et al, 2019 } & 0.0010 \\ \text { Tarekegn AA and Yismaw AE, 20190.0011 } \\ \text { Philips Z,et al, 2003 } & 0.0009 \\ \text { Maharajan MK et al, 2015 } & 0.0001 \\ \text { Kruiroongroj S et al, 2014 (1) } & 0.0013 \\ \text { Kruiroongroj S et al, 2014 (2) } & 0.0004 \\ \text { Alder S et al, 2015 } & 0.0002 \\ \text { Dinh Thu H et al, 2018 } & 0.0015 \\ \text { Umeh IB et al, 2016 } & 0.0004 \\ \text { Philips Z et al, 2006 } & 0.0006 \\ \text { Yan Yuen WW et al, 2018 (1) } & 0.0003 \\ \text { Yan Yuen WW et al, 2018 (2) } & 0.0003 \\ \text { Raab SS et al, 2002 } & 0.0007 \\ \text { Rajiah K et al, 2017 } & 0.0036 \\ \text { Tran BX et al, 2018 } & 0.0023 \\ \text { Touch S and Oh JK, 2018 } & 0.0016 \\ \text { Kristina S et al, 2020 } & 0.0001 \\ & 0.0008\end{array}$

Statistics for each study Lower
limit

0.000

0.0000

0.0000

0.0000

0.0000

0.0000

0.0001

0.0000

0.0001

0.0000

0.0000

0.0000

0.0002

0.0000

0.0001

0.0000

0.0000

0.0000

0.0002

0.0004

0.0002

0.0000

0.0004
Upper
limit

Z-Value

4.1138-

$5.0012-$

4.9595-

4.6928-

3.5682-

4.1796-

4.4559-

1.4734

4.2045-

$4.5904-$

$5.0935-$

1.7043

6.1502-

3.3632-

7.2184

4.6971 -

4.7236-

2.4740-

3.9989 -

$6.4241-$

$5.4075-$

2.3928-

21.1243-
Event rate and $95 \% \mathrm{Cl}$

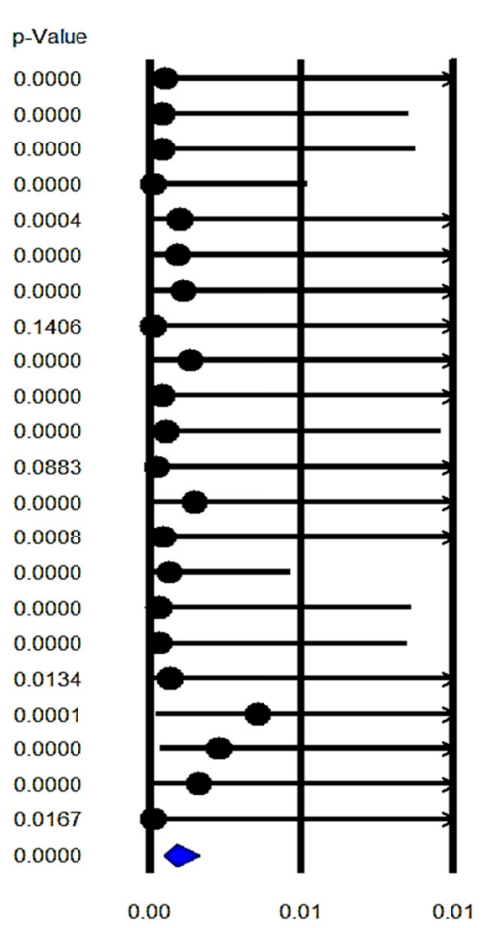

Fig. 5. Meta-analysis of Percentage of Willingness to Pay for Cervical Cancer Prevention Based on GDP per Capita According to Fixed Effect Model with 95\% Confidence Level

Percentage of Willingness to Pay Based on the Gross Domestic Product (GDP) per Capita

Out of the 28 articles included in the study, 18 studies (22 data) were meta-analysis on the percentage of WTP for CCP methods. The results showed that this percentage was $0.84 \%$ (95\% CI 0.44-1.62) (Fig. 5).

Based on GDP per Capita According to Fixed Effect Model with 95\% Confidence Level

The results also showed that among the different groups, the highest percentage of WTP was among the other groups and students; and based on the economic situation of countries, the highest proportion was in LMIEs; and based on the type of prevention methods, the highest percentage was related to the HPV vaccine (Table 3).

The results of the heterogeneous assessment also showed that the results of heterogeneous studies were not consistent and had very good homogeneity $(\mathrm{Q}=6.8 ; \mathrm{d} \mathbf{f}=21$; $\left.\mathrm{I}^{2}=0.00 ; \mathrm{p}<0.98\right)$.

Effective Factors in Acceptance of and Willingness to Pay for Cervical Cancer Prevention Methods

In this study, by analyzing the content of the mentioned factors in the included studies, finally, 31 factors were divided into 2 groups of significant effective factors and nonsignificant effective factors. Most of the factors that have been mentioned in various studies as significant effective factors include income, age, education, risky sexual behaviors, awareness of cervical cancer and its risk factors, belief in cervical cancer risk, and belief about the effectiveness of prevention methods (Table 4).
The Most Important Reasons for Unwillingness to Pay for Cervical Cancer Prevention Methods

Only 7 studies cited reasons for unwillingness to pay for CCP methods. In 6 of the 7 studies, the cost was one of the main reasons cited by indlividuals. Other reasons included lack of information and awareness of preventive methods, difficulties in accessing preventive methods, lack of attention to preventive methods, fear of unsafe methods of prevention, and social stigma.

\section{Reporting Quality Assessment Results}

As most of the reviewed articles were published in highranking journals, most articles complied with reporting assessment items. Out of the 924 assessment items (28 [the number of studies]) multiplied by 33 [number of tool items]), 628 (67.9) items met the standards (the option Yes). About 277 cases (29.9\%) did not comply with the standards (no option) and about 19 cases $(2.2 \%)$ included both "unclear" and "not-applicable" (Table 5).

\section{Discussion}

Most of the reviewed studies were conducted in Southeast Asian countries. Epidemiological studies of cancers, and especially cervical cancer, have also shown that this type of cancer is one of the most common cancer in this region (47). One of the main reasons for this may be the high prevalence of high-risk sexual behaviors and the sex tourism industry in this region, which has been widespread in recent years (48). The results also show a high WTP for and acceptance of CCP in these areas, which is also due to the high prevalence of this type of cancer and 
Table 3. Results of the meta-analysis of the overall acceptance rate, willingness to pay, and percentage of positive willingness to pay based on GDP per capita for cervical cancer prevention methods based on participant variables, countries' economic segregation and types of prevention methods.

\begin{tabular}{|c|c|c|c|c|c|c|c|c|c|}
\hline \multirow{14}{*}{$\begin{array}{l}\text { Acceptance } \\
\text { rate of CCS }\end{array}$} & \multirow[t]{2}{*}{ Variable } & \multirow[t]{2}{*}{ Variable Level } & \multicolumn{3}{|c|}{ Dimension Statues $(95 \% \mathrm{CI})$} & \multicolumn{4}{|c|}{ Heterogeneity Test $(95 \% \mathrm{CI})$} \\
\hline & & & Percent & $\begin{array}{c}\text { Lower } \\
\text { limit }\end{array}$ & $\begin{array}{l}\text { Upper } \\
\text { limit } \\
\end{array}$ & $\overline{\mathrm{df}}$ & $\mathrm{Q}$ & $\mathrm{p}$ & $\mathrm{I}^{2}$ \\
\hline & \multirow[t]{5}{*}{ Participants } & Girls & 48 & 15 & 142 & 1 & 51.58 & 0.000 & 98.061 \\
\hline & & Others & 43 & 25 & 73 & 0 & 0.00 & 1.000 & 0.000 \\
\hline & & Parents/Mothers & 63 & 53 & 76 & 9 & 69.7 & 0.000 & 87.1 \\
\hline & & Students & 62 & 44 & 86 & 4 & 24.5 & 0.000 & 83.7 \\
\hline & & Women & 78 & 63 & 96 & 4 & 20.9 & 0.165 & 80.7 \\
\hline & \multirow{4}{*}{$\begin{array}{l}\text { Country } \\
\text { by economies }\end{array}$} & High-Income Economies (HIEs) & 56 & 40 & 77 & 6 & 119.9 & 0.000 & 94.999 \\
\hline & & $\begin{array}{l}\text { Lower-Middle Income Economies } \\
\text { (LMIEs) }\end{array}$ & 83 & 64 & 108 & 2 & 3.810 & 0.149 & 47.511 \\
\hline & & Low-Income Economies (LIEs) & 88 & 75 & 103 & 0 & 0 & 1 & 1 \\
\hline & & $\begin{array}{l}\text { Upper-Middle-Income Economies } \\
\text { (UMIEs) }\end{array}$ & 63 & 55 & 71 & 11 & 40.4 & 0.000 & 72.8 \\
\hline & \multirow{3}{*}{$\begin{array}{l}\text { Type of prevention } \\
\text { methods }\end{array}$} & $\mathrm{HPV}$ & 60.3 & 53 & 69 & 19 & 175.4 & 0.000 & 89.5 \\
\hline & & Pap-smear & 89.3 & 69 & 115 & 0 & 0.00 & 1.000 & 0.000 \\
\hline & & screening service & 89.5 & 77 & 104 & 0 & 0.25 & 0.61 & 0.000 \\
\hline \multirow{13}{*}{$\begin{array}{l}\text { Rate of Posi- } \\
\text { tive WTP }\end{array}$} & \multirow[t]{6}{*}{ Participants } & Girls & 55 & 46 & 65 & 0 & 0.00 & 1.000 & 0.000 \\
\hline & & Health Professionals & 85 & 67 & 106 & 1 & 0.016 & 0.900 & 0.000 \\
\hline & & Others & 87 & 65 & 115 & 0 & 0.00 & 1.000 & 0.000 \\
\hline & & Parents/Mothers & 67 & 59 & 74 & 7 & 17 & 0.017 & 58.9 \\
\hline & & Students & 87 & 66 & 113 & 1 & 0.00 & 0.942 & 0.000 \\
\hline & & Women & 58 & 40 & 82 & 7 & 137 & 0.000 & 94.9 \\
\hline & \multirow{4}{*}{$\begin{array}{l}\text { Country } \\
\text { by economies }\end{array}$} & High-Income Economies (HIEs) & 65 & 56 & 75 & 5 & 22.4 & 0.000 & 77.7 \\
\hline & & $\begin{array}{l}\text { Lower-Middle Income Economies } \\
\text { (LMIEs) }\end{array}$ & 67 & 53 & 86 & 5 & 15.7 & 0.008 & 68.2 \\
\hline & & Low-Income Economies (LIEs) & 73 & 54 & 96 & 2 & 6 & 0.04 & 66.9 \\
\hline & & $\begin{array}{l}\text { Upper-Middle-Income Economies } \\
\text { (UMIEs) }\end{array}$ & 64 & 43 & 96 & 6 & 13.7 & 0.00 & 95.6 \\
\hline & \multirow{3}{*}{$\begin{array}{l}\text { Type of prevention } \\
\text { methods }\end{array}$} & HPV & 63 & 52 & 76 & 15 & 197.2 & 0.000 & 92.3 \\
\hline & & Pap-smear & 89 & 69 & 115 & 0 & 0.0 & 1.000 & 0.000 \\
\hline & & screening service & 71 & 61 & 83 & 4 & 7.1 & 0.1 & 43.7 \\
\hline \multirow{13}{*}{$\begin{array}{l}\text { WTP as a } \\
\text { percentage of } \\
\text { GDP per } \\
\text { capita }\end{array}$} & \multirow[t]{6}{*}{ Participants } & Girls & 0.19 & 0 & 0.24 & 1 & 0.1 & 0.733 & 0.000 \\
\hline & & Health Professionals & 1 & 011 & 9.1 & 1 & 0.0 & 0.937 & 0.000 \\
\hline & & Others & 2.27 & 0.35 & 14.3 & 0 & 0.0 & 1.000 & 0.000 \\
\hline & & Parents/Mothers & 0.40 & 0.10 & 1.51 & 6 & 0.0 & 1.000 & 0.000 \\
\hline & & Students & 1.78 & 0.29 & 10.5 & 3 & 0.5 & 0.904 & 0.000 \\
\hline & & Women & 0.94 & 0.31 & 2.09 & 5 & 1.01 & 0.96 & 0.000 \\
\hline & \multirow{4}{*}{$\begin{array}{l}\text { Country } \\
\text { by economies }\end{array}$} & High-Income Economies (HIEs) & 0.39 & 0.13 & 1.19 & 8 & 0.7 & 0.999 & 0.000 \\
\hline & & $\begin{array}{l}\text { Lower-Middle Income Economies } \\
\text { (LMIEs) }\end{array}$ & 1.54 & 0.49 & 4.79 & 4 & 0.91 & 0.91 & 0.000 \\
\hline & & Low-Income Economies (LIEs) & 1 & 0.1 & 9.12 & 1 & 0.0 & 0.937 & 0.000 \\
\hline & & $\begin{array}{l}\text { Upper-Middle-Income Economies } \\
\text { (UMIEs) }\end{array}$ & 1 & 0.26 & 4.13 & 5 & 1.4 & 0.923 & 0.000 \\
\hline & \multirow{3}{*}{$\begin{array}{l}\text { Type of prevention } \\
\text { methods }\end{array}$} & HPV & 0.91 & 0.43 & 1.90 & 16 & 505 & 0999 & 0.000 \\
\hline & & Pap-smear & 0.53 & 0.02 & 1.14 & 1 & 0.00 & 0936 & 0.000 \\
\hline & & screening service & 0.63 & 0.12 & 3.3 & 2 & 0.27 & 0.87 & 0.000 \\
\hline
\end{tabular}

its association with high-risk sexual behaviors. Also, one of the factors contributing to the high WTP and acceptance of CCP in these areas could be the issue of high economic growth in the countries in the region in recent years (49).

The HPV vaccine was the most common type of prevention methods in most studies. HPV accounts for $90 \%$ of cervical cancer cases $(50,51)$. The WHO recommends the HPV vaccine be included in countries' vaccination programs (52). However, in the present study, the HPV vaccine had a lower WTP and acceptance rate compared to other methods and had a high percentage of GDP per capita. However, Numerous other studies' results have confirmed the superiority and advantages of the HPV vaccine in comparison with other methods $(53,54)$. One of the main reasons for this issue may be the novelty of this method, as the HPV vaccine has been introduced and used since 2006, while other methods have been used for many years. The results of this study showed that the cost of prevention is one of the main reasons for the unwillingness or acceptance of CCP methods. Therefore, it is recommended that different countries and organizations adopt cost-cutting methods by adopting supportive policies, such as insurance coverage.

In this study, although CCP methods accounted for a high percentage of GDP per capita in LMICs, the rates of acceptance and WTP in these countries were higher than HICs. One of the main reasons for this may be the high prevalence of cervical cancer in LMICs. Studies have estimated that about $85 \%$ of the prevalence and mortality of this type of cancer occur in LMICs (55). However, these countries have many problemsi with these types of diseases and usually have very limited potential and resources for the prevention and treatment of these diseases $(56,57)$. An important reason for the high payment amount of GDP per capita in these countries is the low per capita GDP. Therefore, more attention should be paid to the policies and strategies offered by various organizations, especially 


\begin{tabular}{|c|c|c|c|c|}
\hline \multicolumn{2}{|c|}{ Variable } & \multirow{2}{*}{$\frac{\text { Significant positiv }}{* * * * * * * * * * *}$} & & Nonsignificant \\
\hline 1. & Household Income & & & $* * *$ \\
\hline 2. & Age & $* * * * * * * * * * *$ & & $* * *$ \\
\hline 3. & Age of daughters & $* *$ & & \\
\hline 4. & Test status (had screening test or not) & $* *$ & & $* *$ \\
\hline 5. & Had heard of screening test & $* * * *$ & & $* *$ \\
\hline 6. & Had heard of cervical cancer and risk factors & $* * * * *$ & & $* *$ \\
\hline 7. & Had heard of HPV vaccine & $*$ & & \\
\hline 8. & Perception on the health of daughters & * & & \\
\hline 9. & Risky sexual behaviors & $* * * * *$ & & $* * * * * *$ \\
\hline & Education & $* * * * * * * * * *$ & & $* * * *$ \\
\hline 11. & Self-rated health & & & $* * *$ \\
\hline 12. & perceived seriousness of cervical cancer & $* * * *$ & & $* *$ \\
\hline 13. & perceived quality /accuracy of screening test & $* *$ & & $*$ \\
\hline 14. & Marital status & $* * * * *$ & & $* * *$ \\
\hline & Religion & & & $*$ \\
\hline & Ethnicity & & & $* *$ \\
\hline 17. & Background profession & & & $* *$ \\
\hline 18. & Knowledge about screening test & $* * *$ & & \\
\hline 19. & Source of more Information about screening test & $* *$ & & * \\
\hline 20. & Proportion of screening testing normal & $*$ & & \\
\hline 21. & Smoking & $*$ & & * \\
\hline & Has risk factor (STDs, family history...) & $* * *$ & & \\
\hline & employment & $* *$ & & \\
\hline & No. of children & $* * *$ & & $* * *$ \\
\hline & Believes screening test is an effective & $* * * *$ & & $* * *$ \\
\hline & Living setting (urban or rural) & ** & & $* *$ \\
\hline & Being local residents & $*$ & & \\
\hline & Having daughter(s) & $*$ & & \\
\hline & Family history of cancer & ** & & \\
\hline & Private insurance status & $*$ & & \\
\hline & Knowledge & $*$ & & \\
\hline \multicolumn{5}{|c|}{ *Number/ frequency } \\
\hline \multicolumn{5}{|c|}{ Table 5. Example of Quality Assessment of Included Studies } \\
\hline & Question & & $\begin{array}{l}\text { Wordsworth S, } \\
\text { et al: } 2001\end{array}$ & $\begin{array}{l}\text { Choi HCW } \\
\text { et al, } 2013\end{array}$ \\
\hline 1 & (a) Indicate the study's design with a commonly used term in the title or the abstract & & Yes & Yes \\
\hline 2 & $\begin{array}{l}\text { (b) Provide in the abstract an informative and balanced summary of what was do } \\
\text { found }\end{array}$ & e and what was & Yes & Yes \\
\hline 3 & Explain the scientific background and rationale for the investigation being reported & & Yes & Yes \\
\hline 4 & State specific objectives, including any prespecified hypotheses & & Yes & Yes \\
\hline 5 & Present key elements of study design early in the paper & & Yes & Yes \\
\hline 6 & $\begin{array}{l}\text { Describe the setting, locations, and relevant dates, including periods of recruitme } \\
\text { low-up, and data collection }\end{array}$ & exposure, fol- & Yes & Yes \\
\hline 7 & (a) Give the eligibility criteria, and the sources and methods of selection of participa & & Yes & Yes \\
\hline 8 & $\begin{array}{l}\text { Clearly define all outcomes, exposures, predictors, potential confounders, and effec } \\
\text { diagnostic criteria, if applicable }\end{array}$ & modifiers. Give & Yes & Yes \\
\hline 9 & $\begin{array}{l}\text { For each variable of interest, give sources of data and details of methods of asse } \\
\text { ment). Describe comparability of assessment methods if there is more than one grou }\end{array}$ & ment (measure- & Yes & Yes \\
\hline 10 & Describe any efforts to address potential sources of bias & & No & No \\
\hline 11 & Explain how the study size was arrived at & & Yes & Yes \\
\hline 12 & $\begin{array}{l}\text { Explain how quantitative variables were handled in the analyses. If applicable, descr } \\
\text { ings were chosen and why }\end{array}$ & which group- & Yes & Yes \\
\hline 13 & (a) Describe all statistical methods, including those used to control for confounding & & Yes & Yes \\
\hline 14 & (b) Describe any methods used to examine subgroups and interactions & & No & No \\
\hline 15 & (c) Explain how missing data were addressed & & No & Yes \\
\hline 16 & (d) If applicable, describe analytical methods taking account of sampling strategy & & Yes & Yes \\
\hline 17 & (e) Describe any sensitivity analyses & & No & No \\
\hline 18 & (b) Give reasons for non-participation at each stage & & No & No \\
\hline 19 & $\begin{array}{l}\text { (a) Give characteristics of study participants (eg demographic, clinical, social) an } \\
\text { exposures and potential confounders }\end{array}$ & information on & Yes & Yes \\
\hline 20 & (b) Indicate number of participants with missing data for each variable of interest & & No & No \\
\hline
\end{tabular}

by the WHO, to better control of this type of cancer and to increase the effectiveness of prevention methods $(58,59)$.

In terms of the significant factors in acceptance and WTP, in addition to the issues related to the costs and risky sexual behaviors mentioned, most of the other factors were related to individuals' knowledge and attitude (such as education, cervical cancer awareness, and its risk factors, etc.). Many studies have been published in recent years about the knowledge and attitude of screening and prevention methods of cervical cancer $(60,61)$. The results of most studies indicate that people's awareness, especially women about cervical cancer are not enough and prevention methods and interventions are needed to increase their awareness. Therefore, due to the low 


\begin{tabular}{|c|c|c|c|}
\hline \multicolumn{4}{|c|}{ Table 5. Ctd } \\
\hline & Question & $\begin{array}{c}\text { Wordsworth S, } \\
\text { et al: } 2001\end{array}$ & $\begin{array}{l}\text { Choi HCW } \\
\text { et al, } 2013 \\
\end{array}$ \\
\hline 21 & Report numbers of outcome events or summary measures & Yes & Yes \\
\hline 22 & $\begin{array}{l}\text { (a) Give unadjusted estimates and, if applicable, confounder-adjusted estimates and their precision } \\
\text { (eg, } 95 \% \text { confidence interval). Make clear which confounders were adjusted for and why they were } \\
\text { included }\end{array}$ & Yes & Yes \\
\hline 23 & (b) Report category boundaries when continuous variables were categorized & Yes & Yes \\
\hline 24 & Summarise key results with reference to study objectives & Yes & Yes \\
\hline 25 & $\begin{array}{l}\text { Discuss limitations of the study, taking into account sources of potential bias or imprecision. Discuss } \\
\text { both direction and magnitude of any potential bias }\end{array}$ & Yes & Yes \\
\hline 26 & $\begin{array}{l}\text { Give a cautious overall interpretation of results considering objectives, limitations, multiplicity of } \\
\text { analyses, results from similar studies, and other relevant evidence }\end{array}$ & Yes & Yes \\
\hline 27 & Discuss the generalisability (external validity) of the study results & Yes & Yes \\
\hline 28 & $\begin{array}{l}\text { Give the source of funding and the role of the funders for the present study and, if applicable, for the } \\
\text { original study on which the present article is based }\end{array}$ & No & Yes \\
\hline 29 & Does a detailed description of goods or services in question offered to the respondents? & No & Yes \\
\hline 30 & $\begin{array}{l}\text { Does the information and attributes expressed in goods or services scenarios is obtained from user or } \\
\text { key informant assessments (e.g. focus groups, Delphi panels, interviews etc)? }\end{array}$ & No & Yes \\
\hline 31 & Was there a pilot study conducted to assess the survey tool/design? & No & No \\
\hline 32 & Does the survey involve face to face interviews? & No & No \\
\hline 33 & Were those involved in data collection adequately trained? & No & NA \\
\hline
\end{tabular}

knowledge of individuals and the impact of attention given to these issues in acceptance and WTP for screening and prevention of cervical cancer, there is a need to increase public awareness in this regard.

Based on the results of the present study, it is suggested that policymakers and senior managers of the health system of countries, while considering the local conditions of their country, in general, pay attention to reducing the cost of preventive measures, to increase awareness, and improve people's attitudes about prevention methods, and to focus on target age groups and appropriate prevention and reducing risky sexual behaviors to increase the WTP and acceptance of prevention methods.

However, based on the results of the literature review and the experiences of the researchers, the present study for the first time systematically and comprehensively examined the different dimensions of WTP for and acceptance of different CCP methods and provided comprehensive and applicable information to policymakers and researchers. However, during this study, the researchers faced several limitations, one of which was the restriction of the articles to the CVM. In this study, only published studies in English and Persian were reviewed. Also, due to the type of results reporting, the authors could not conduct meta-analyses based on some of the variables, especially the type of information provided to participants that is a key first step in using the CVM method.

\section{Conclusion}

The results showed that the WTP for and acceptance of CCP methods was relatively high among individuals. According to the results of this study it is recommended that attention be paid to reduce the cost of preventive measures, especially the HPV vaccine, to increase awareness and to improve people's attitudes about prevention methods, to focus on target age groups and appropriate prevention, and to reduce risky sexual behaviors to increase the WTP and acceptance of CCP methods. Also, it is recommended to consider other methods of calculating WTP and other cancers in future studies.

\section{Acknowledgement}

This study was part of a $\mathrm{PhD}$ thesis in Health Economics at Iran University of Medical Sciences (IUMS), supported by Iran University of Medical Sciences (Grant No: IUMS/SHMIS_98-2-37-15495 and with Ethical code: IR.IUMS.REC.1398.423).

\section{Ethical Approval}

The thesis was approved by an Ethics approval code: IR.IUMS.REC.1398.423.

\section{Conflict of Interests}

The authors declare that they have no competing interests.

\section{References}

1. Pedersen K, Burger EA, Nygard M, Kristiansen IS, Kim JJ. Adapting cervical cancer screening for women vaccinated against human papillomavirus infections: The value of stratifying guidelines. Eur J Cancer. 2018;91:68-75.

2. Baudendistel I, Winkler EC, Kamradt M, Brophy S, Langst G, Eckrich $\mathrm{F}$, et al. Cross-sectoral cancer care: views from patients and health care professionals regarding a personal electronic health record. Eur J Cancer Care. 2016;3(10):12429.

3. Mathers CD, Loncar D. Projections of global mortality and burden of disease from 2002 to 2030. PLoS Med. 2006;3(11):e442.

4. Murphy KM, Nguyen V, Shin K, Sebastian-Deutsch A, Frieden L. Health Care Professionals and the Employment-Related Needs of Cancer Survivors. J Occup Rehabil. 2016;8:8.

5. Bahr S, Bzieh R, El Hayek GY, Adib S. Cost-benefit analysis of a projected national human papilloma virus vaccination programme in Lebanon. East Mediterr Health J. 20119;25(10):715-21.

6. Anorlu RI. Cervical cancer: the sub-Saharan African per-spective. Reprod Health Matters. 2008;16(32)):41-9.

7. Sawaya GF, Smith-Mccune K, Kuppermann M. Cervical Cancer Screening: More Choices in 2019. JAMA. 2019;321(20):2018-9.

8. Smith A, Baines N, Memon S, Fitzgerald N, Chadder J, Politis C, et al. Moving toward the elimination of cervical cancer: Modelling the health and economic benefits of increasing uptake of human papillomavirus vaccines. Curr Oncol. 2019;26(2):80-4.

9. Li S, Liu S, Ratcliffe J, Gray A, Chen G. Preferences for cervical cancer screening service attributes in rural China: a discrete choice experiment. Patient Prefer Adherence. 2019;13:881-9.

10. Azami-Aghdash S, Gharaee H, Aghaei MH, Derakhshani N Cardiovascular Disease Patient's Quality of Life in Tabriz City in Iran 
in 2018. J Community Health Res. 2019.

11. Lin YL, Lin Z, He F, Hu ZJ, Zimet GD, Alias H, et al. Factors influencing intention to obtain the HPV vaccine and acceptability of 24-and 9-valent HPV vaccines: A study of undergraduate female health sciences students in Fujian, China. Vaccine. 2019;37(44):6714-23.

12. O'Brien B. Cost-Benefit Analysis, Willingness to Pay. Wiley StatsRef: Statistics Reference Online. 2014.

13. Mariani AW, Pêgo-Fernandes PM. Willingness to pay... What??? Sao Paulo Med J. 2014;132(3):131-2.

14. Azami-Aghdash S, Sadeghi-Bazargani H, Saadati M, Mohseni M, Gharaee H. Experts' perspectives on the application of public-private partnership policy in prevention of road traffic injuries. Chin $\mathrm{J}$ Traumatol. 2020;23(3):152-158.

15. Boyle KJ. Contingent valuation in practice. A primer on nonmarket valuation: Springer; 2017. p. 83-131.

16. Umeh IB, Nduka SO, Ekwunife OI. Mothers' willingness to pay for HPV vaccines in Anambra state, Nigeria: a cross sectional contingent valuation study. Cost Eff Resour Alloc. 2016;14:8.

17. Philips Z, Johnson S, Avis M, Whynes DK. Human papillomavirus and the value of screening: young women's knowledge of cervical cancer. Health Educ Res. 2003;18(3):318-28.

18. Rajiah K, Maharajan MK, Chin NS, Num KS. Awareness and acceptance of human papillomavirus vaccination among health sciences students in Malaysia. Virusdisease. 2015;26(4):297-303.

19. Khan K, Kunz R, Kleijnen J, Antes G. Systematic reviews to support evidence-based medicine: Crc Press; 2011.

20. Moher D, Shamseer L, Clarke M, Ghersi D, Liberati A, Petticrew M, et al. Preferred reporting items for systematic review and metaanalysis protocols (PRISMA-P) 2015 statement. Syst Rev. 2015;4(1):1.

21. Weng Q, Jiang J, Haji FM, Nondo LH, Zhou H. Women's knowledge of and attitudes toward cervical cancer and cervical cancer screening in Zanzibar, Tanzania: a cross-sectional study. BMC Cancer. 2020;20(1):63.

22. Wordsworth S, Ryan M, Waugh N. Costs and benefits of cervical screening IV: valuation by women of the cervical screening programme. Cytopathology. 2001;12(6):367-76.

23. Choi HCW, Leung GM, Woo PPS, Jit M, Wu JT. Acceptability and uptake of female adolescent HPV vaccination in Hong Kong: A survey of mothers and adolescents. Vaccine. 2013;32(1):78-84.

24. Tarekegn AA, Mengistu MY, Mirach TH. Health professionals' willingness to pay and associated factors for cervical cancer screening program at College of Medicine and Health Sciences, University of Gondar, Northwest Ethiopia. PLoS One. 2019;14(4):12.

25. Tarekegn AA, Yismaw AE. Health professionals' willingness to pay and associated factors for human papilloma virus vaccination to prevent cervical cancer at College of Medicine and Health Sciences University of Gondar, Northwest Ethiopia. BMC Res Notes. 2019;12(1):58.

26. Hoque ME, Ghuman S, Van Hal G. Human Papillomavirus Vaccination Acceptability among Female University Students in South Africa. Asian Pac J Cancer Prev. 2013;14(8):4865-9.

27. Maharajan MK, Rajiah K, Num KS, Yong NJ. Knowledge of Human Papillomavirus Infection, Cervical Cancer and Willingness to pay for Cervical Cancer Vaccination among Ethnically Diverse Medical Students in Malaysia. Asian Pac J Cancer Prev. 2015;16(14):5733-9.

28. Kruiroongroj S, Chaikledkaew U, Thavorncharoensap M. Knowledge, Acceptance, and Willingness to Pay for Human Papilloma Virus (HPV) Vaccination among Female Parents in Thailand. Asian Pac J Cancer Prev. 2014;15(13):5469-74.

29. Songthap A, Pitisuttithum P, Kaewkungwal J, Fungladda W, Bussaratid V. Knowledge, attitudes, and acceptability of a human papilloma virus vaccine among students, parents and teachers in Thailand. Southeast Asian J Trop Med Public Health. 2012;43(2):34053.

30. Alder S, Gustafsson S, Perinetti C, Mints M, Sundstrom K, Andersson S. Mothers' acceptance of human papillomavirus (HPV) vaccination for daughters in a country with a high prevalence of HPV. Oncol Rep. 2015;33(5):2521-8.

31. Dinh Thu H, Nguyen Thanh H, Hua Thanh T, Nguyen Hai L, Tran Thi V, Nguyen Manh T, et al. Mothers' willingness to pay for daughters' HPV vaccine in northern Vietnam. Health Care Women Int. 2018;39(4):450-62.

32. Philips Z, Whynes DK, Avis M. Testing the construct validity of willingness to pay valuations using objective information about risk and health benefit. Health Econ. 2006;15(2):195-204.

33. Yan Yuen WW, Lee A, Chan PKS, Tran L, Sayko E. Uptake of human papillomavirus (HPV) vaccination in Hong Kong: Facilitators and barriers among adolescent girls and their parents. PLoS One. 2018;13(3).

34. Liao CH, Liu JT, Pwu RF, You SL, Chow I, Tang CH. Valuation of the economic benefits of human prapillomavirus vaccine in Taiwan. Value Health. 2009;12 Suppl 3:S74-7.

35. Raab SS, Grzybicki DM, Hart AR, Kiely S, Andrew-JaJa C, Scioscia E, Jr. Willingness to pay for new Papanicolaou test technologies. Am J Clin Pathol. 2002;117(4):524-33.

36. Dahlström LA, Tran TN, Lundholm C, Young C, Sundström K, Sparén P. Attitudes to HPV vaccination among parents of children aged 12-15 years-A population-based survey in Sweden. Int J Cancer. 2010;126(2):500-7.

37. Oh JK, Lim MK, Yun EH, Lee EH, Shin HR. Awareness of and attitude towards human papillomavirus infection and vaccination for cervical cancer prevention among adult males and females in Korea: A nationwide interview survey. Vaccine. 2010;28(7):1854-60.

38. Rajiah K, Maharajan MK, Num KSF, Koh RCH. Knowledge about human papillomavirus and cervical cancer: predictors of HPV vaccination among dental students. Asian Pac J Cancer Prev. 2017;18(6):1573.

39. Tran BX, Than PTQ, Doan TTN, Nguyen HLT, Mai HT, Nguyen THT, et al. Knowledge, attitude, and practice on and willingness to pay for human papillomavirus vaccine: a cross-sectional study in Hanoi, Vietnam. Patient Prefer Adherence. 2018;12:945.

40. Touch S, Oh JK. Knowledge, attitudes, and practices toward cervical cancer prevention among women in Kampong Speu Province, Cambodia. BMC Cancer. 2018;18(1).

41. Opoku CA, Browne ENL, Spangenberg K, Moyer C, Kolbilla D, Gold KJ. Perception and risk factors for cervical cancer among women in northern Ghana. Ghana Med J. 2016;50(2):84-9.

42. Lin Y, Lin Z, He F, Chen H, Lin X, Zimet GD, et al. HPV vaccination intent and willingness to pay for 2-, 4-, and 9-valent HPV vaccines: A study of adult women aged $27-45$ years in China. Vaccine. 2020.

43. You D, Han L, Li L, Hu J, Zïmet GD, Alias H, et al. Human Papillomavirus (HPV) Vaccine Uptake and the Willingness to Receive the HPV Vaccination among Female College Students in China: A Multicenter Study. Vaccines (Basel). 2020;8(1):31.

44. Lin W, Wang Y, Liu Z, Chen B, Yuan S, Wu B, et al. Inequalities in Awareness and Attitude towards HPV and Its Vaccine between Local and Migrant Residents Who Participated in Cervical Cancer Screening in Shenzhen, China. Cancer Res Treat. 2020;52(1):207-17.

45. Kristina S, Darmawan K, Sandly N, Worojati T. Perception of seriousness, knowledge, and willingness to pay for cervical cancer screening among women in Indlonesia. Int Medical J. (1994). 2020;25(4):2019-26.

46. Bank TW. World Bank Country and Lending Groups 2019 [Available from: https://datahelpdesk.worldbank.org/knowledgebase/ articles/906519.

47. Lancaster KE, Lungu T, Bula A, Shea JM, Shoben A, Hosseinipour $\mathrm{MC}$, et al. Preferences for Pre-exposure Prophylaxis Service Delivery Among Female Sex Workers in Malawi: A Discrete Choice Experiment. AIDS Behav. 2019.

48. Arbyn M, Weiderpass E, Bruni L, de Sanjosé S, Saraiya M, Ferlay J, et al. Estimates of incidence and mortality of cervical cancer in 2018: a worldwide analysis. Lancet Glob Health. 2020;8(2):e191-e203.

49. Hooi DJ, Quint WG, Lissenberg-Witte BI, Kenter G, Pinedo HM, de Koning MN, et al. Human papillomavirus (HPV) types prevalence in cervical samples of female sex-workers on Curaçao. Prev Med Rep. 2018;11:120-4.

50. Santhanes D, Wong CP, Yap YY, San SP, Chaiyakunapruk N, Khan TM. Factors involved in humanı papillomavirus (HPV) vaccine hesitancy among women in the South-East Asian Region (SEAR) and Western Pacific Region (WPR): a scoping review. Hum Vaccin Immunother. 2018;14(1):124-33.

51. Bong A, Premaratne G. Regional integration and economic growth in Southeast Asia. Glob Bus Rev. 2018;19(6):1403-15.

52. Hill H. Southeast Asia in the global economy: a selective analytical survey. Philipp Rev Econ. 2018.

53. Organization WH. The selection and use of essential medicines: report of the WHO expert committee, 2015 (including the 19th WHO model list of essential medicines and the 5th WHO model list of 
essential medicines for children): World Health Organization; 2015.

54. FAAEM F. Tarascon Pocket Pharmacopoeia 2017 Deluxe Lab-Coat Edition: Jones \& Bartlett Learning; 2016.

55. Altobelli E, Scarselli G, LattanzI A, Fortunato C, Profeta VF. A comparison between Pap and HPV screening tests and screening methods. Mol Clin Oncol. 2016;5(2):348-54.

56. Randall TC, Ghebre R. Challenges in prevention and care delivery for women with cervical cancer in sub-Saharan Africa. Front Oncol. 2016;6:160.

57. Chuang L, Temin S, Camacho R, Dueñas-Gonzalez A, Feldman S, Gultekin M. Management and Care of Women With Invasive Cervical Cancer: American Society of Clinical Oncology Resource-Stratified Clinical Practice Guideline. J Glob Oncol. 2016;2:311-40.

58. Small Jr W, Bacon MA, Bajaj A, Chuang LT, Fisher BJ, Harkenrider MM, et al. Cervical cancer: a global health crisis. Cancer. 2017;123(13):2404-12.

59. LaVigne AW, Triedman SA, Randall TC, Trimble EL, Viswanathan AN. Cervical cancer in low and middle income countries: Addressing barriers to radiotherapy delivery. Gynecol Oncol Rep. 2017;22:16-20.

60. Canfell K, Kim JJ, Brisson M, Keane A, Simms KT, Caruana M, et al. Mortality impact of achieving WHO cervical cancer elimination targets: a comparative modelling analysis in 78 low-income and lower-middle-income countries. Lancet. 2020;395(10224):591-603.

61. Thapa N, Maharjan M, Petrini MA, Shah R, Shah S, Maharjan N, et al. Knowledge, attitude, practice and barriers of cervical cancer screening among women living in mid-western rural, Nepal. J Gynecol Oncol. 2018;29(4). 


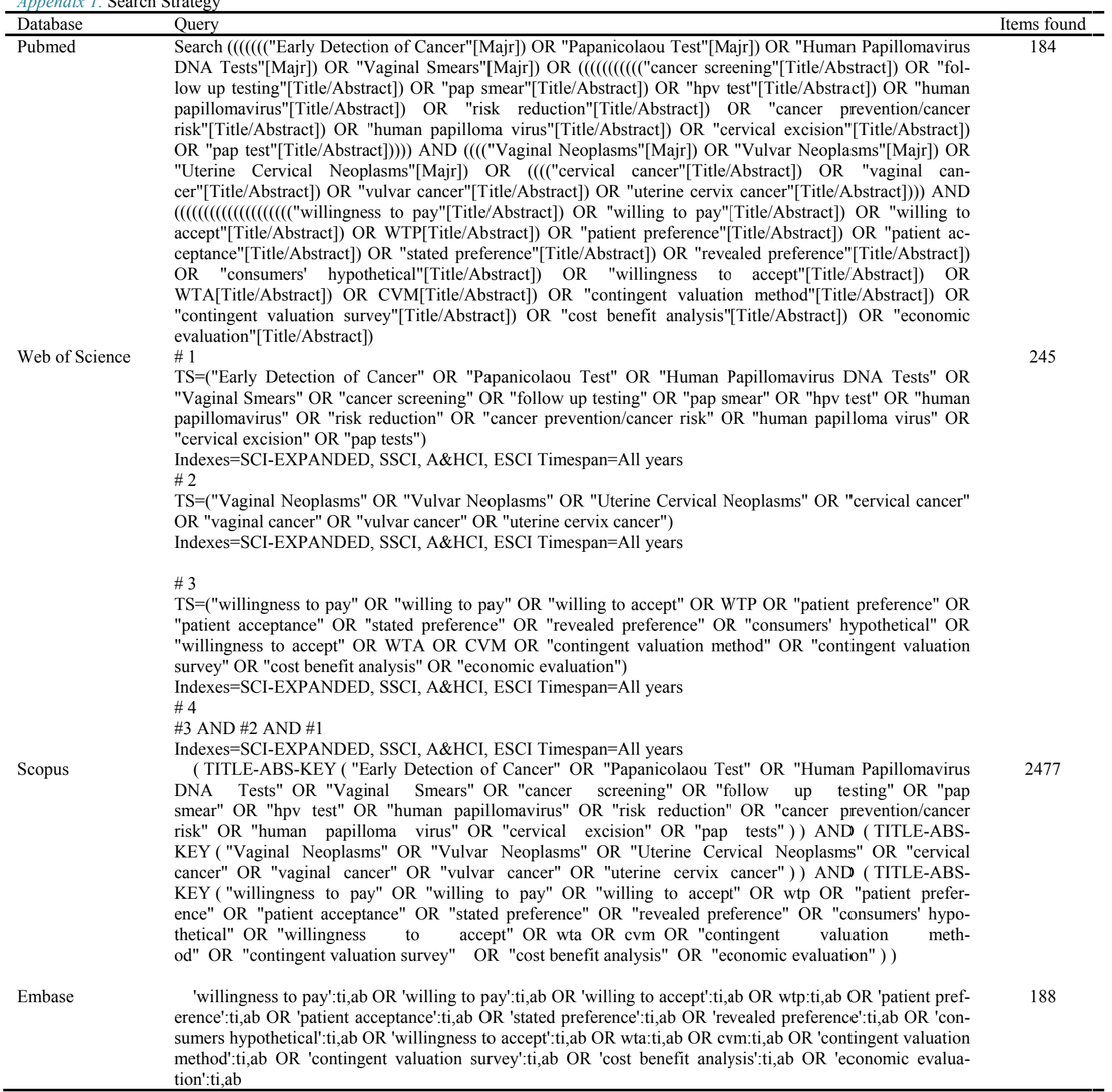

